\title{
Inference of cell-type specific imprinted regulatory elements and genes during human neuronal differentiation
}

Dan Liang ${ }^{1,2}$, Nil Aygün ${ }^{1,2}$, Nana Matoba ${ }^{1,2}$, Folami Y. Ideraabdullah ${ }^{1}$, Michael I. Love ${ }^{1,3}$, Jason L. Stein ${ }^{1,2,4}$

1) Department of Genetics, University of North Carolina at Chapel Hill, Chapel Hill, NC 27599, USA

2) UNC Neuroscience Center, University of North Carolina at Chapel Hill, Chapel Hill, NC 27599, USA

3) Department of Biostatistics, University of North Carolina at Chapel Hill, Chapel Hill, NC 27599, USA

4) Lead Contact: jason_stein@med.unc.edu

Running title: Inference of neural genomic imprinting 


\section{ABSTRACT}

Genomic imprinting results in gene expression biased by parental chromosome of origin and occurs in genes with important roles during human brain development. However, the cell-type and temporal specificity of imprinting during human neurogenesis is generally unknown. By detecting within-donor allelic biases in chromatin accessibility and gene expression that are unrelated to cross-donor genotype, we inferred imprinting in both primary human neural progenitor cells (phNPCs) and their differentiated neuronal progeny from up to 85 donors. We identified 43/20 putatively imprinted regulatory elements (IREs) in neurons/progenitors, and 133/79 putatively imprinted genes in neurons/progenitors. Though 10 IREs and 42 genes were shared between neurons and progenitors, most imprinting was only detected within specific cell types. In addition to well-known imprinted genes and their promoters, we inferred novel IREs and imprinted genes. We found IREs overlapped with $\mathrm{CpG}$ islands more than non-imprinted regulatory elements. Consistent with DNA methylation-based regulation of imprinted expression, some putatively imprinted regulatory elements also overlapped with differentially methylated regions on the maternal germline. Finally, we identified a progenitor-specific putatively imprinted gene overlap with copy number variation that is associated with uniparental disomy-like phenotypes. Our results can therefore be useful in interpreting the function of variants identified in future parent-of-origin association studies.

\section{INTRODUCTION}

In contrast to most loci in the genome that have roughly equal expression from either parental chromosome, genomic imprinting leads to biased levels of gene expression or chromatin accessibility from either the maternal or paternal chromosome. Some genomic imprinting results 
in totally silenced expression of one parental allele and is more likely to be shared across multiple tissues (Bonthuis et al. 2015). However, most imprinted genes exhibit some tissue and cell-type specific imprinted expression (Bonthuis et al. 2015; Kravitz and Gregg 2019; Zink et al. 2018). Some genes are imprinted specifically in humans (Nakabayashi et al. 2011) and many imprinted genes are also expressed in neural development or in the adult brain (Babak et al. 2015; Barlow and Bartolomei 2014; Perez, Rubinstein, and Dulac 2016). Previous studies have identified imprinted genes in different human tissues or cells (Babak et al. 2015; Baran et al. 2015; Santoni et al. 2017), but imprinted regulatory elements (IREs) have not been well defined during human neurogenesis nor has their cell-type specificity been assessed. Elucidation of cell-type specific imprinting mechanisms during neurogenesis are critical for interpreting results of parent-of-origin association studies for neuropsychiatric disorders and subsequent therapeutic development (Perez, Rubinstein, and Dulac 2016; Ishida and Moore 2013; Nicholls 2000; Mozaffari et al. 2019; Brandler et al. 2018; Wolter et al. 2020).

The primary regulatory elements (REs) that control genomic imprinting, called imprinting control elements (ICEs) or imprinting control regions (ICRs), exhibit parental specific DNA methylation. Some of these differentially methylated regions (DMRs) are inherited from the sperm or egg and are maintained postfertilization throughout development in all tissues (Ferguson-Smith 2011; Hanna and Kelsey 2014; Arnaud 2010; Plasschaert and Bartolomei 2014). A separate class of DMRs acquire methylation after fertilization under the direction of germ-cell specific DMRs and show tissue-specific methylation patterns (Lopes et al. 2003; Lucifero et al. 2002). Cell-type specific "reading" of germline DMRs and other epigenetic alterations such as histone modifications without associated germ-cell specific DMRs also contribute to regulation of imprinted expression in specific tissues/cell-types (Prickett and Oakey 2012; Arnaud 2010; 
Andergassen et al. 2017; Xu Wang, Soloway, and Clark 2011; Q. Wang et al. 2011). However, these tissue/cell-type specific regulatory elements controlling imprinting are not well identified.

Genomic imprinting is most often studied in mice where reciprocal cross breeding is designed, parents are genotyped, and parent-of-origin specific gene expression is measured in offspring using high-throughput sequencing data (X. Wang and Clark 2014; Oreper et al. 2018; Crowley et al. 2015). Heterozygous genetic markers then allow the separation of maternal vs paternal expression (Babak et al. 2008; Xu Wang et al. 2008; Gregg et al. 2010; Laukoter et al. 2020). These studies have found that there are more genes with imprinted expression in the brain as compared to non-brain tissues (Perez et al. 2015) and that genomic imprinting shows key functions in neurodevelopmental processes including neural progenitor expansion, migration, differentiation, and cell polarization in mouse brains (Perez, Rubinstein, and Dulac 2016). However, in humans, it is difficult to obtain both molecular phenotypes and genotype data from children and genotype data from both parents on a large scale in order to demonstrate genomic imprinting. It is still possible to infer imprinting expression in humans where parental genotypes are unavailable by detecting within donor allelic imbalance unrelated to across donor genotype in large datasets where sequencing data for gene expression/chromatin accessibility and genotyping have been collected (Figure 1A) (Reinius and Sandberg 2015; Baran et al. 2015). Here, we used the beta-binomial distribution to model allelic counts across a population up to 85 genetically diverse donors, in order to estimate the dispersion of the allelic ratio (AR; reference allelic count to total count) in the population, for gene expression (RNA-seq) and chromatin accessibility (ATAC-seq). Higher dispersion of the allelic ratio is suggestive of parentally biased expression or accessibility as the parental allele is not expected to be consistent with reference allele coding. Using this method, we identify putatively IREs and genes in human neural progenitors and neurons. 


\section{RESULTS}

\section{Inference of IREs and genes in progenitors and neurons}

We utilized primary human neural progenitor (phNPC) chromatin accessibility and expression quantitative trait loci (QTL) datasets described in our previous work (Liang et al. 2021; Aygün et al. 2021) to infer imprinted genes and REs. phNPCs were cultured in vitro as progenitor cells and also differentiated for 8 weeks, virally labeled, and sorted to obtain a homogeneous population of neurons. We then performed ATAC-seq and RNA-seq to obtain chromatin accessibility profiles (Liang et al. 2021) (N_Progenitor=76 and N_Neuron=61) and gene expression profiles (Aygün et al. 2021) (N_Progenitor=85 and N_Neuron=74) in both cell types (Figure 1A). We identified heterozygous genetic variants using imputed genotype data for all donors in progenitors and neurons in order to distinguish the two chromosomes, though we are unable to classify maternal or paternal origin. Allele-specific chromatin accessibility and gene expression were then calculated using read counts (for the reference allele and the alternative allele) at each accessible/expressed heterozygous single nucleotide polymorphism (SNP) site. We identified 19,960 heterozygous SNPs in 12,233 accessible regions and 80,811 expressed heterozygous SNPs in 11,770 genes in neurons; and 42,706 heterozygous SNPs in 10,802 accessible regions and 63,733 expressed heterozygous SNPs in 8,958 genes in progenitors.

The silenced imprinted allele, inherited from one parent, will by definition lead to decreased chromatin accessibility/expression as compared to the imprinted expressed allele. Here, we do not know the parental origin of each allele at a heterozygous locus. We expect that at an imprinted site, the AR will be either high (when the reference allele is the expressed imprinted allele) or low (when the reference allele is the silenced imprinted allele) leading to high dispersion in estimates

of the AR across donors. We estimated the dispersion of allelically biased chromatin accessibility or gene expression to identify the SNPs with highly variable (either high or low) AR in the 
population. We modeled allelic counts of chromatin accessibility or gene expression using a betabinomial distribution at heterozygous SNPs (Skelly et al. 2011; Castel et al. 2015) and evaluated the significance of the over-dispersion of AR using a likelihood ratio test. SNPs in known randomly monoallelic expression (RMAE) genes and randomly monoallelic chromatin accessible (RMACA) regions were removed (Xu et al. 2017; Gimelbrant et al. 2007). Finally, we kept SNPs with a significant AR over-dispersion (FDR $<0.05$ across all heterozygous SNPs tested within a given cell type (Benjamini and Hochberg 1995)). A minimal donor count for both high and low AR ( $\mathrm{n}_{\text {donor }}$ $\geq 2$ for $A R \geq 0.8$ and $n_{\text {donor }} \geq 2$ for $A R \leq 0.2$ ) was used to exclude cases of allele specific expression or chromatin accessibility, i.e. AR driven by genetic effects (Figure 1A). Because our study lacks parental genotype data, we cannot exclude the possibility that allelic bias in newly identified imprinted genes/REs is due to previously unidentified RMAE or RMACA rather than parental inheritance. Thus, we refer to these allele-specifically expressed genes/REs as putatively imprinted.

After these analyses and filtering steps, we identified 43 IREs containing 57 SNPs in neurons (nIREs) and 20 IREs containing 25 SNPs in progenitors (pIREs) (Figure 1B-1C; Supplementary Figure 1A, 1C and 1D; Supplemental Table S1). We found 3 nIREs overlapped with known human ICEs for known imprinted genes PEG10, MEST and ZIM2/PEG3 (Cowley et al. 2018), providing confidence in IRE calls. We also found 10 shared nIREs and pIREs overlapped with the promoters of 11 well-known imprinted genes (Supplemental Figure 1A; red labeled points) that are involved in neuronal development and differentiation: MAGEL2, NDN, SGCE, PEG10, NAA60, MIMT1, ZNF597, MEST, PEG3, ZIM2 and SNRPN (Watrin et al. 2005; Grütz et al. 2017; Ono et al. 2006; Babak et al. 2015; Nakabayashi et al. 2011). Surprisingly, we did not find any pIREs that overlapped with the promoters of well-known imprinted genes, so the pIREs identified here may 
represent a novel set of imprinted elements, such as the promoters of EIF2D, DDX11L2 and SPEG.

We identified 133 neuron imprinted genes (20 of which are previously known imprinted genes) containing 653 SNPs and 79 progenitor imprinted genes (15 of which are previously known imprinted genes) containing 166 SNPs (Figure 1B-1C; Supplemental Figure 1B; Supplemental Table S2). For these imprinted genes, many genes have been previously reported as imprinted, such as UBE3A, NDN, PEG3, MEST, GRB10 and MEG3 (Dindot et al. 2008; Huntriss et al. 2013; Blagitko et al. 2000; Zhang et al. 2003; Jay et al. 1997; Ho-Shing and Dulac 2019). Imprinted genes like $D L K 1$ and $Z D B F 2$ have previously described important functions in corticogenesis (Duffié et al. 2014; Bouschet et al. 2017; Ferrón et al. 2011; Surmacz et al. 2012). We also found novel imprinted genes specifically in neurons, such as HM13, ZNF331, COPG2, DOC2B and PBX1 (Supplemental Table S2) that have not been previously described as imprinted but where the expression patterns fit the characteristics of imprinting. However, we could not detect all of the known imprinted genes, such as IGF2, due to lack of expression in these two cell types (GTEx Consortium 2020; Nowakowski et al. 2017).

We found 42 genes that show imprinting patterns in both progenitors and neurons (Figure 1B, Supplementary Table 1), including the well-known imprinted genes: GRB10, PEG10, MEST, MEG3, MEG8, NDN, and SNHG14. Several of the imprinted genes that are shared across cell types have not been previously identified as imprinted, such as PIANP and SNHG7. We identified 10 REs showing evidence of imprinting in both progenitors and neurons, at the promoters of EIF2D, DDX11L2, IL17RC, CRELD1, FAM86B3P, FAM86GP, FAM86FP, PIP4P1, EEF2KMT and ZNF826P. Many of these REs overlapped with promoters of genes that are not previously known as imprinted genes, such as CRELD1 and EIF2D, which could be new candidates for imprinting regulation. Though there was some overlap of imprinting between cell types 
(31.6\%/53.2\% imprinted genes and $23.3 \% / 50.0 \%$ IREs in neurons/progenitors), most imprinting was found only in one cell type. Often this was because the gene did not survive QC in both cell types (Supplementary Figure 1C and 1D). Nevertheless, cell type specific imprinting patterns were still detectable when the same gene passed QC in both cell types. Overall, the detection of well-known imprinted genes and regulatory elements supports the statistical approach to identify imprinted candidates outlined here. Novel genes and regulatory elements may have been undetected in previous studies due to the lack of a cell type specific or development system for studying these effects. 
Figure 1
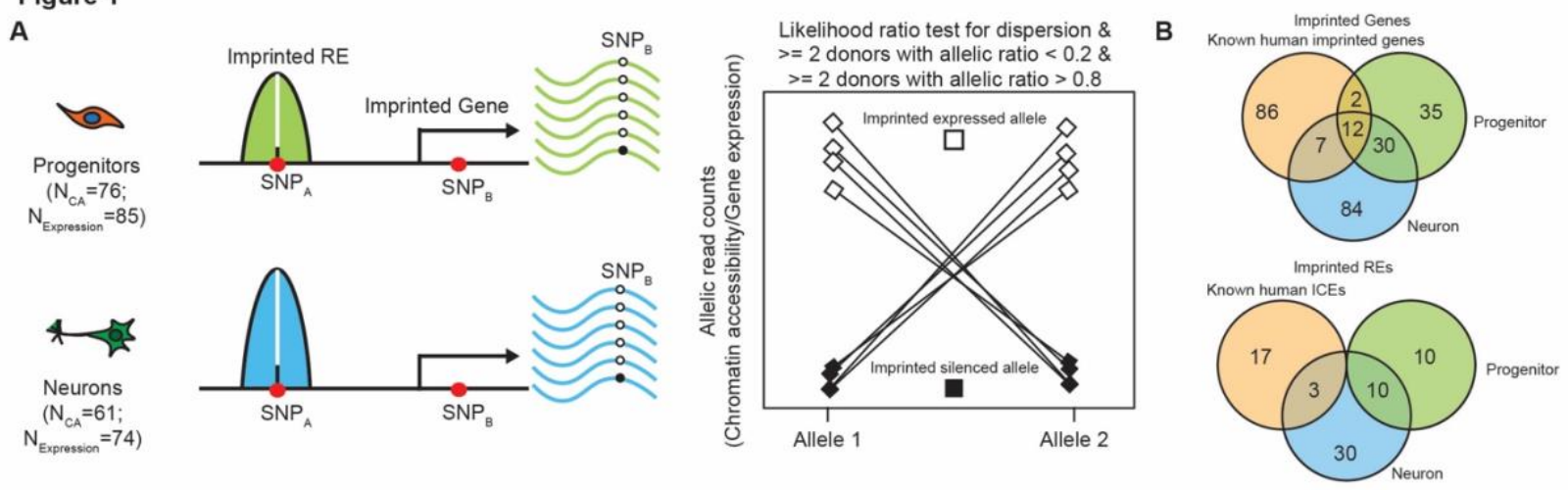

c

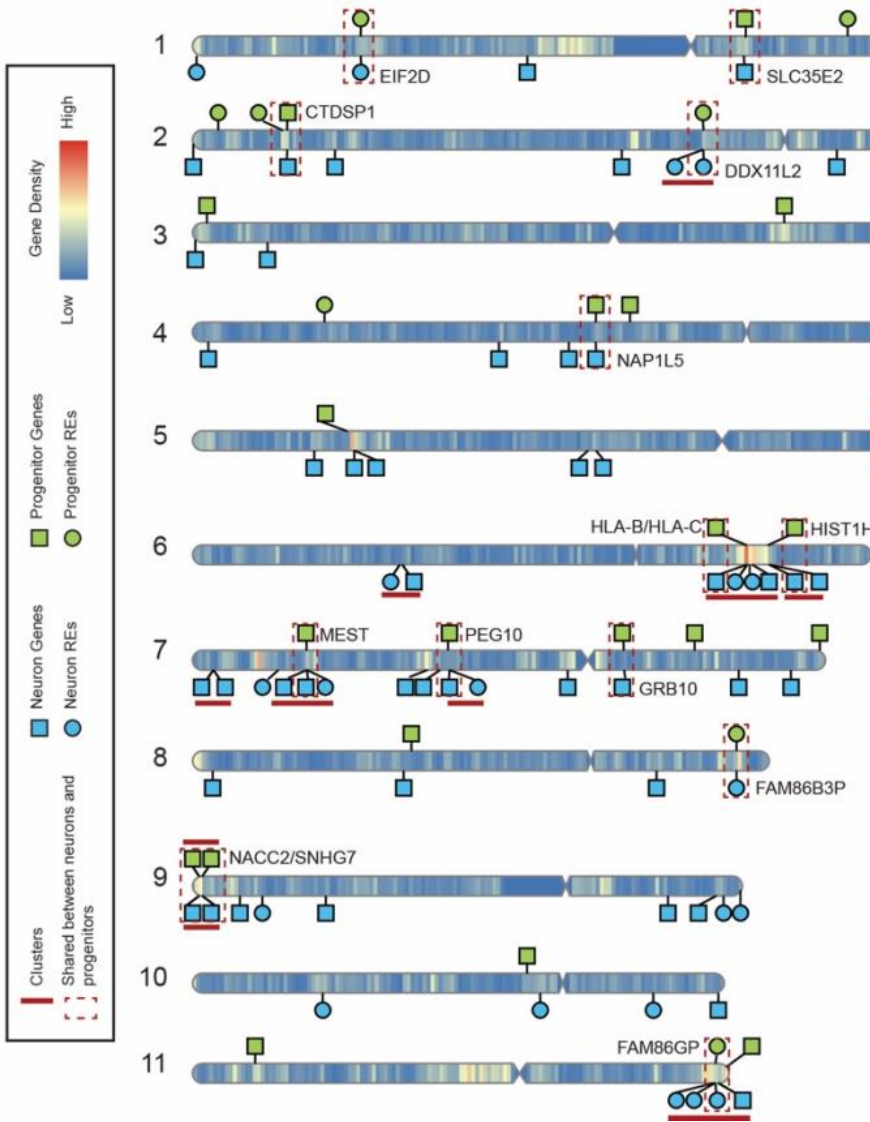

Figure 1. Identification of neuron/progenitor imprinted genes and Res. (A)Schematic cartoon of experimental design and methods.(B) Comparison of imprinted genes and REs in neurons, progenitors and known imprinted genes/ICEs.(C) Ideogram of neuron/progenitor imprinted genes and REs on the human genome. 
bioRxiv preprint doi: https://doi.org/10.1101/2021.10.04.463060; this version posted October 12, 2021. The copyright holder for this preprint (which was not certified by peer review) is the author/funder, who has granted bioRxiv a license to display the preprint in perpetuity. It is made available under aCC-BY 4.0 International license.

\section{Supplemental Figure 1}
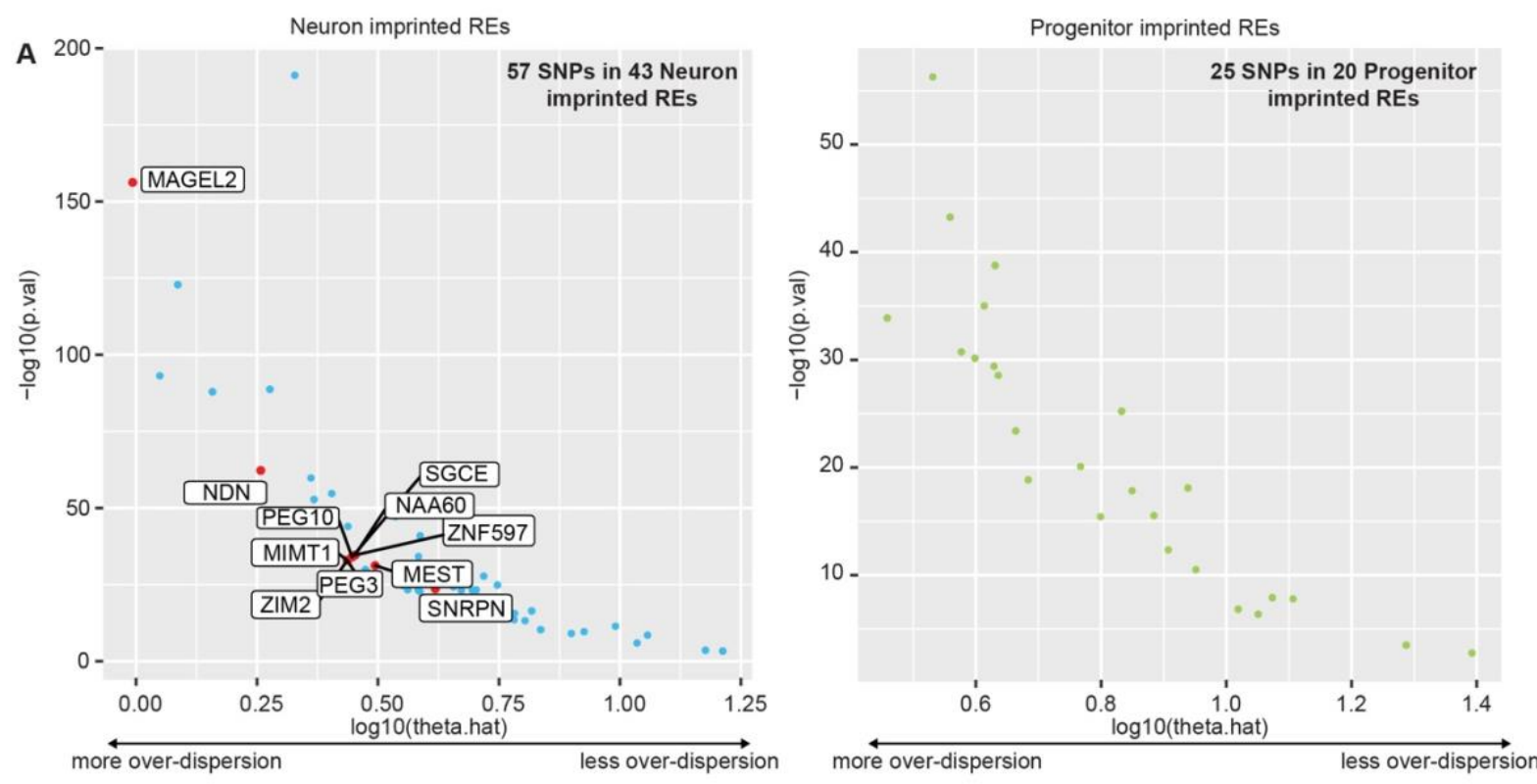

B

Neuron imprinted Genes

Progenitor imprinted Genes
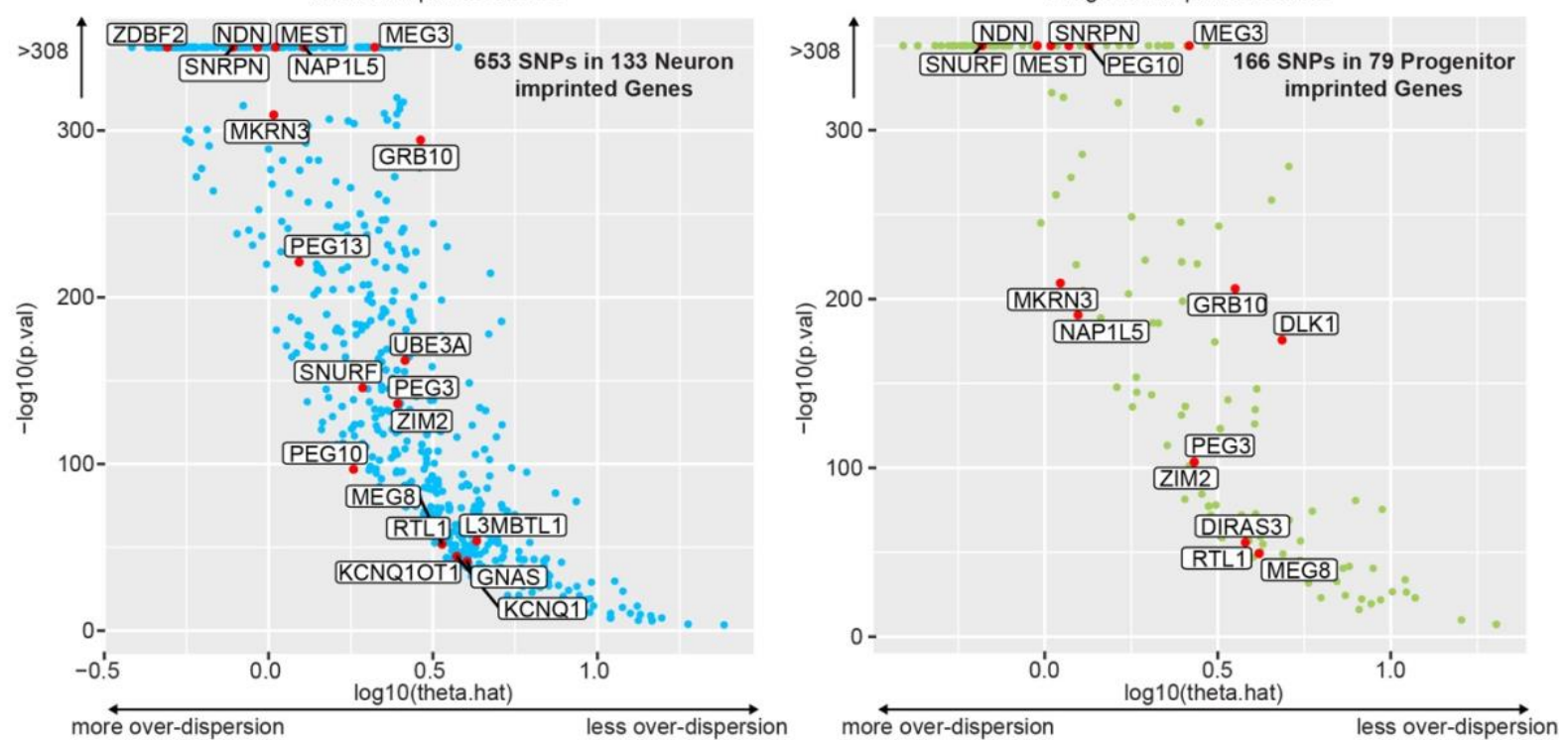

C Number of heterozygous SNPs in

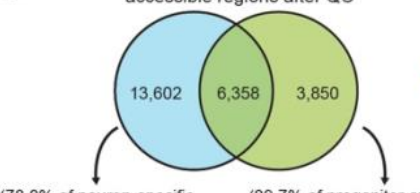

(78.0\% of neuron-specific
SNPs in neuron peaks) (89.7\% of progenitor-specific
SNPs in progenitor

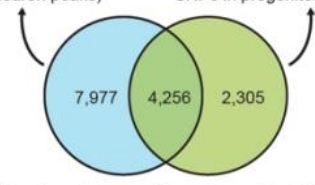

Number of accessible regions after $\mathrm{QC}$ $(42.9 \% / 46.1 \%$ of shared SNPs in progenitor/neuron peaks)

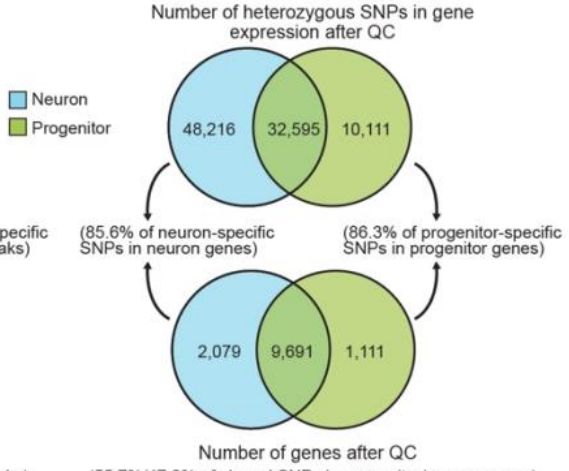

( $55.7 \% / 47.2 \%$ of shared SNPs in progenitor/neuron genes)
D $\square \square \begin{aligned} & \text { Evidence for imprinting in neurons/progenitors } \\ & \text { (QC not passed in progenitors/neurons) }\end{aligned}$

$\square \square$ Evidence for imprinting in neurons/progen-

Evidence for imprinting in neurons
and progenitors
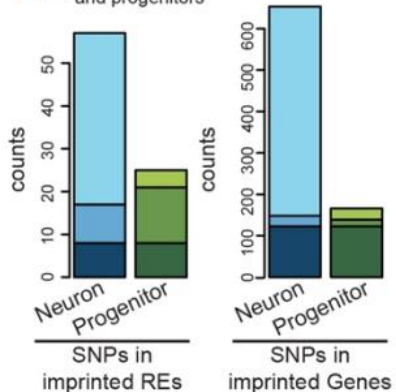
Supplemental Figure 1. (A) Dispersion level and the $p$ values for SNPs in IREs. IREs overlapping with the promoters of known imprinted genes are labeled by red dots. (B) Dispersion level and the $p$ values for SNPs in imprinted genes. (C) Comparison of SNPs in gene expression region and REs (before and after $Q C$ ) between neurons and progenitors. (D) Comparison of SNPs in imprinted gene expression region and REs between neurons and progenitors.

\section{Neuron/progenitor imprinting at known loci}

A well-known example of an imprinted genomic cluster is the Prader-Willi/Angelman Syndrome (PWS/AS) region on human chromosome 15q11-q13 (Nicholls, Saitoh, and Horsthemke 1998). Mutations in the PWS/AS region result in neurodevelopmental disorders, PWS and AS (Perk et al. 2002), in a parent-of-origin dependent manner, demonstrating the important function of these imprinted genes during neural development. We identified neuron-specific IREs that overlap with the promoters of MAGEL2, NDN and SNRPN, the latter with multiple SNPs supporting the imprinting inference (Figure 1C, $2 \mathrm{~A}$ and $2 \mathrm{~B}$ ). 72 SNPs in UBE3A passed our filtering criteria and showed evidence for neuron-specific imprinting of gene expression (Figure 2A and 2C; Supplemental Figure 2A). This finding is in agreement with previous studies showing that UBE3A is expressed exclusively from the maternally inherited allele in neurons (Martins-Taylor et al. 2014; Hsiao et al. 2019). In the PWS/AS region, we found more IREs and genes in neurons than in progenitors, which is also consistent with previous studies in iPSC-derived neurons (PólvoraBrandão et al. 2018; Stanurova et al. 2016). 
Figure 2

A

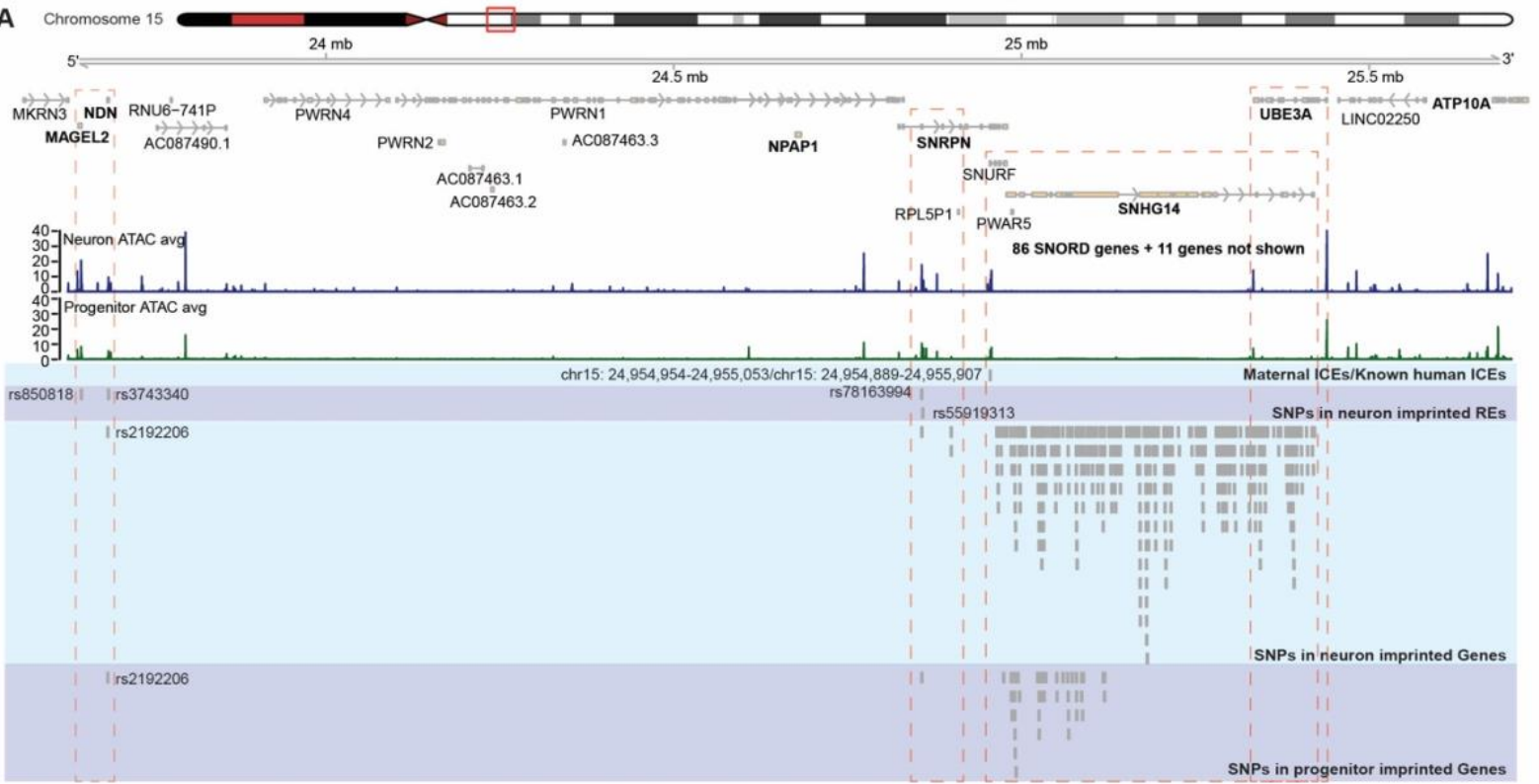

B

Neuron MAGEL2 promoter Pvalue $=5.518 \mathrm{e}-157$

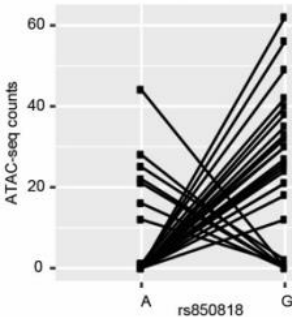

C

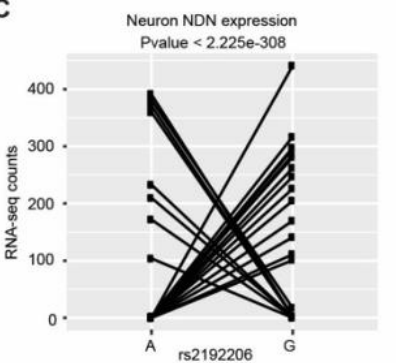

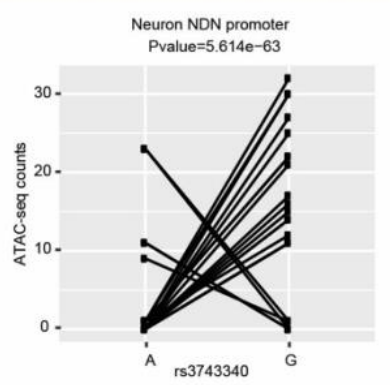

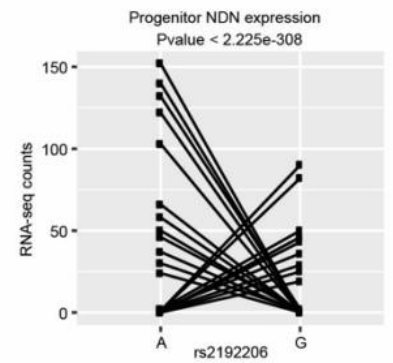

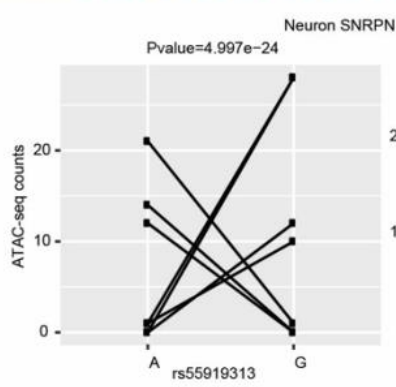
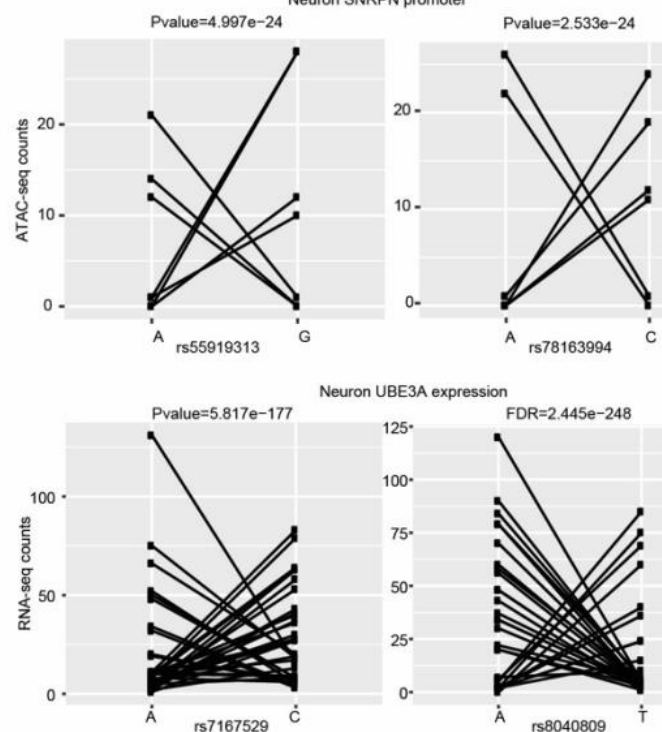

Neuron UBE3A expr

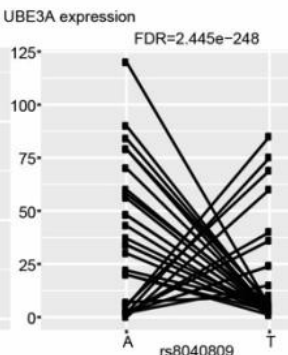

Figure 2. Imprinted genes and REs at known genomic imprinting loci. (A) Coverage plot of ATAC-seq in neurons and progenitors at the AS/PWS lous and SNPs in the imprinted REs and genes. (B) Allelic ATAC-seq counts for selected SNPs in imprinted REs in (A). (C) Allelic RNA-seq counts for selected SNPs in imprinted genes in (A). 


\section{Supplemental Figure 2}

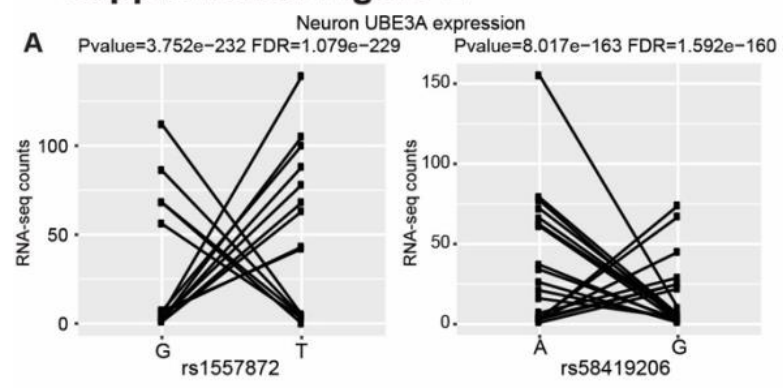

Deuron ZNF
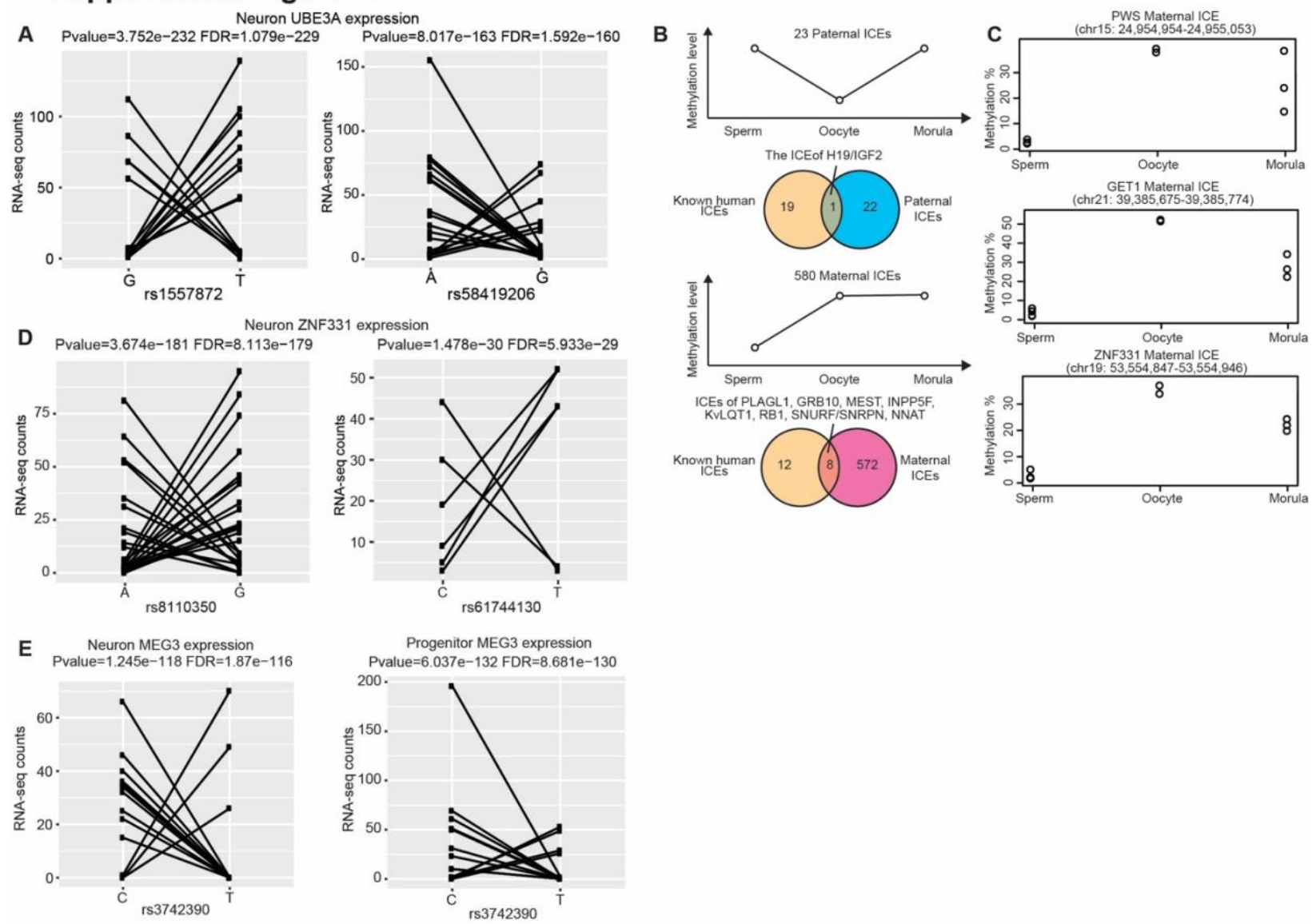

Supplemental Figure 2. (A) Allelic ATAC-seq counts for the SNPs in the expression region of UBE3A. (B) Schematic cartoon of paternal (23) and maternal (580) ICEs and overlaps of ICEs and promoters of known imprinted genes. (C) Methylation rates of ICEs in sperm, oocytes and morula. (D) Allelic ATAC-seq counts for the SNPs in the expression region of ZNF331. (E) Allelic ATAC-seq counts for the SNPs in the expression region of MEG3.

\section{Methylation and transcription factor binding at IREs}

The methylation of $\mathrm{CpG}$ sites is an important epigenetic regulation of imprinting in mammals (Paulsen and Ferguson-Smith 2001). To explore the relationship between methylation and IREs in neurons and progenitors, we first calculated the GC content of the IREs. We found the GC content is significantly higher within IREs as compared to non-imprinted REs (Figure 3A). We found the IREs showed significantly higher overlap with human CpG islands than the nonimprinted REs in both neurons and progenitors (Figure 3B). Enrichment of CpG sites at IREs 
supports a role for DNA methylation in genomic imprinting at these loci during neuronal differentiation.

DNA-binding proteins (DBP) binding to the IREs have important roles in maintaining imprinting by changing the methylation levels around their binding sites during early development (Takahashi et al. 2019; Sanli and Feil 2015). Additionally, methylation at IREs may block some DBPs binding on the imprinted silenced allele thereby decreasing expression of genes from the imprinted silenced allele. However, it is unclear which DBPs are involved in either maintaining imprinting or the downstream consequences of imprinting during human neurogenesis, especially at cell-type resolution. To identify the DBPs that may bind to and regulate IREs in neurons and progenitors, we performed an enrichment analysis of transcription factor (TF) motifs in the IREs using a binomial test (McLean et al. 2010). We retained only the TFs with significantly higher expression in the cell type tested for enrichment (Figure 3C). Among TF motifs enriched in neuron IREs, we found ELK4, which is involved in upstream regulation of parent-of-origin-regulated genes in mice with sleep loss (Tinarelli et al. 2014). We also found that CTCF TF motifs were enriched in pIREs. CTCF was shown to regulate the imprinted expression of $K L D 1$ by binding to the ICE at $K L D 1$ MEG3 locus in embryo stem cells (Llères et al. 2019). Using these IREs, we are able to identify TFs implicated in imprinting gene regulation during human neurogenesis. 
Figure 3
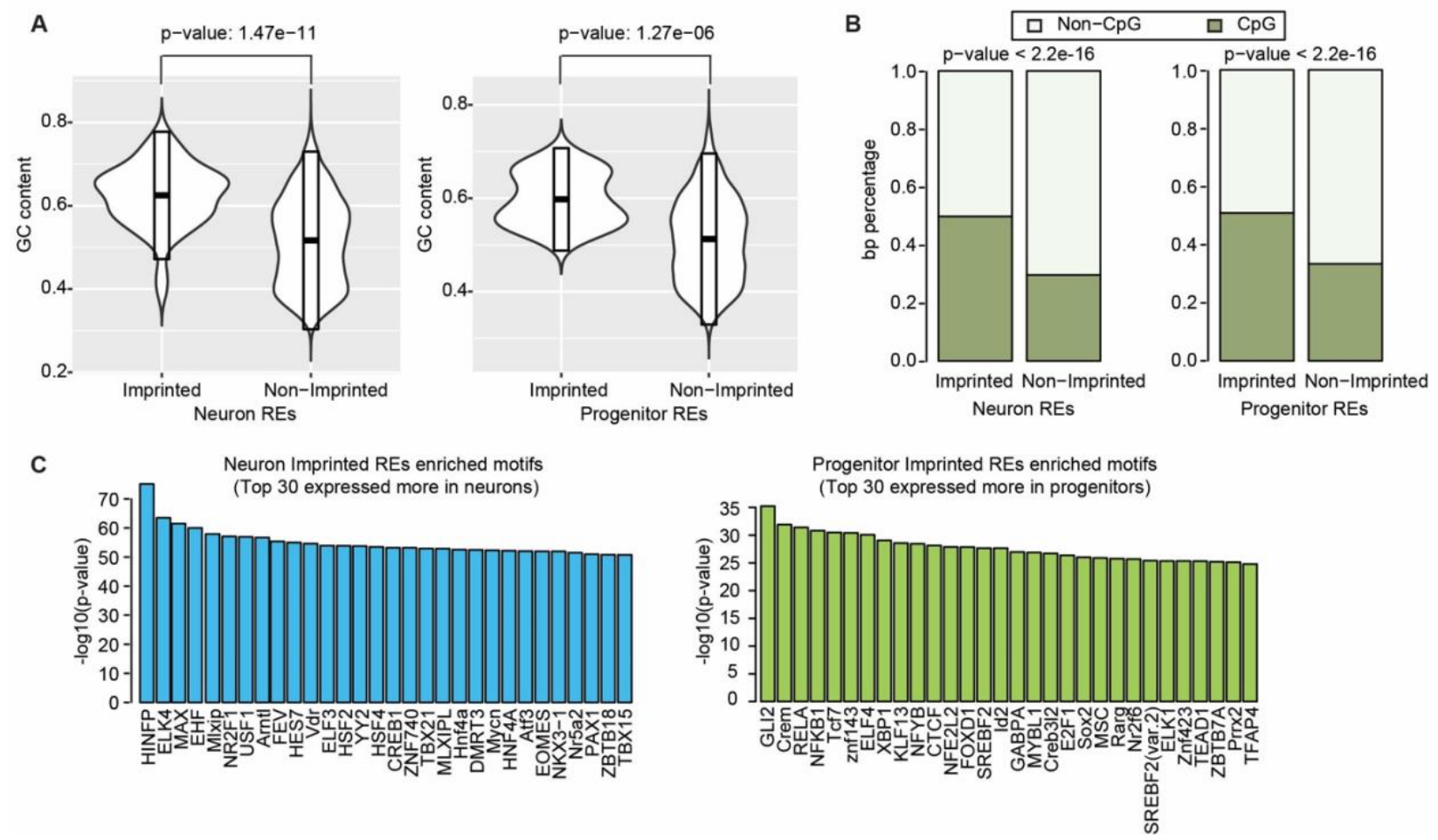

Figure 3. Methylation and transcription factor binding at imprinted REs. (A) GC content of imprinted and non-imprinted REs in neurons (left) and progenitors (right). (B) Overlap of imprinted and non-imprinted REs with human CpG islands in neurons (left) and progenitors (right). (C) Enriched TF motifs within imprinted REs as compared to non-imprinted REs.

\section{Imprinted REs and genes indicate isoform-specific imprinting in neurons}

Previous studies showed isoform-specific imprinted transcription for well known genes, like PEG1 and MEST (Kosaki et al. 2000; Stelzer et al. 2015). However, the number of isoform-specific imprinted genes may still be underestimated, because isoform expression is highly cell type specific and imprinting has not been assessed in all cell types. Using IREs in neurons and progenitors, we predicted isoform-specific imprinted expression in each cell type. In neurons, we found that the promoter region (chr21:39,385,651-39,386,540) of one isoform (ENST00000380713) of the gene GET1 (also known as WRB) showed an imprinting pattern at two SNPs (Figure 4A and 4B). We did not find any SNP passed QC in this RE in progenitors, 
therefore we are not able to test if this RE is a pIRE. This region in WRB was previously reported as a new candidate imprinted region according to DNA methylation analysis using peripheral blood samples (Docherty et al. 2014; Alves da Silva et al. 2016), but to our knowledge, this is the first time this region was suggested as an IRE in neurons. WRB is a receptor associated with protein transmembrane transport (Vilardi, Lorenz, and Dobberstein 2011); but, its function in the human brain is unknown. This region overlapped with a differentially methylated region that is more methylated in oocyte and morula than sperm and maintains methylation in fetal and adolescent brain (Figure 4C; Supplementary Figure 2B-C) (Guo et al. 2014; Lister et al. 2013). The DMR inherited from germ cells in the soma provides evidence that this IRE is an ICE that is maintained throughout development and potentially regulates maternal genomic imprinting of a specific GET1 isoform. No SNPs in the expression level data survived QC so we could not test whether GET1 isoform expression was imprinted (Plasschaert and Bartolomei 2014; Hanna and Kelsey 2014).

We also found the gene ZNF331 had cell-type and isoform-specific imprinted expression patterns (Figure 4D and 4E; Supplementary Figure 2D). We found a neuron-specific IRE (chr19:53,537,591-53,538,420) overlapped with the promoter of a subset of isoforms of ZNF331 and these isoforms showed neuron-specific imprinted expression patterns (Figure 4E, boundaries of the imprinted isoforms are indicated by blue arrows). We also found a germline DMR near the promoters of the isoforms that could serve as an ICE of this region (Supplementary Figure 2C-D; Guo et al. 2014). The germline DMR is more methylated in oocyte and morula as compared to sperm (Supplementary Figure 2D), suggesting maternal imprinting. However, in progenitors, SNPs in this locus did not pass the threshold for significance and/or AR, so it was not possible to test for imprinted expression. Notably, isoform-specific imprinting of ZNF331 was previously reported in multiple cell types and tissues, including the brain and LCLs (boundaries of LCL imprinted isoforms are indicated by red arrows in Figure 4D) (Court et al. 2014; Jadhav et al. 
bioRxiv preprint doi: https://doi.org/10.1101/2021.10.04.463060; this version posted October 12, 2021. The copyright holder for this preprint (which was not certified by peer review) is the author/funder, who has granted bioRxiv a license to display the preprint in perpetuity. It is made available under aCC-BY 4.0 International license.

2019; Daelemans et al. 2010; Ben-David, Shohat, and Shifman 2014). These results indicate celltype specific allelically biased isoform expression during neuronal development and differentiation. 
bioRxiv preprint doi: $h t t p s: / / d o i . o r g / 10.1101 / 2021.10 .04 .463060 \cdot$ this version posted October 12,2021 The copyright holder for this preprint (which was not certified by peer review) is the author/funder, who has granted bioRxiv a license to display the preprint in perpetuity. It is made available under aCC-BY 4.0 International license.

Figure 4

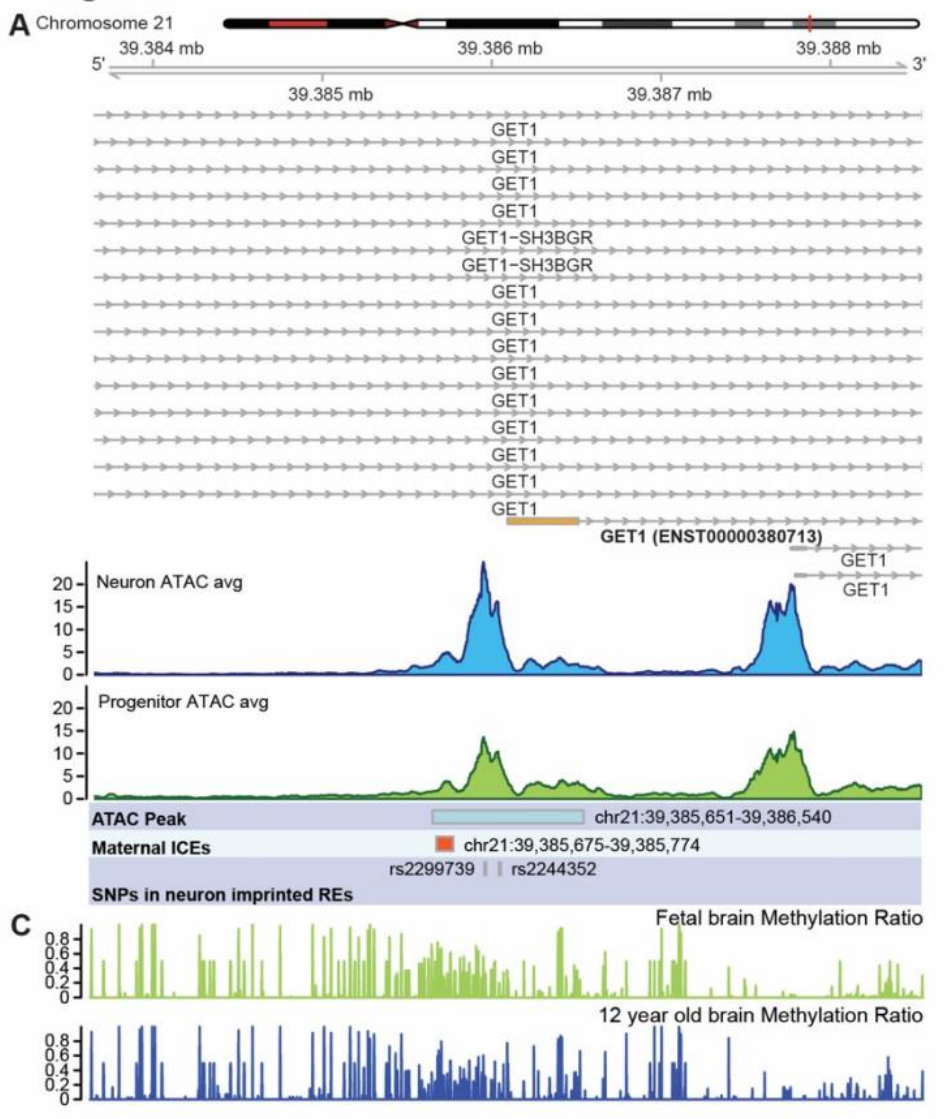

D

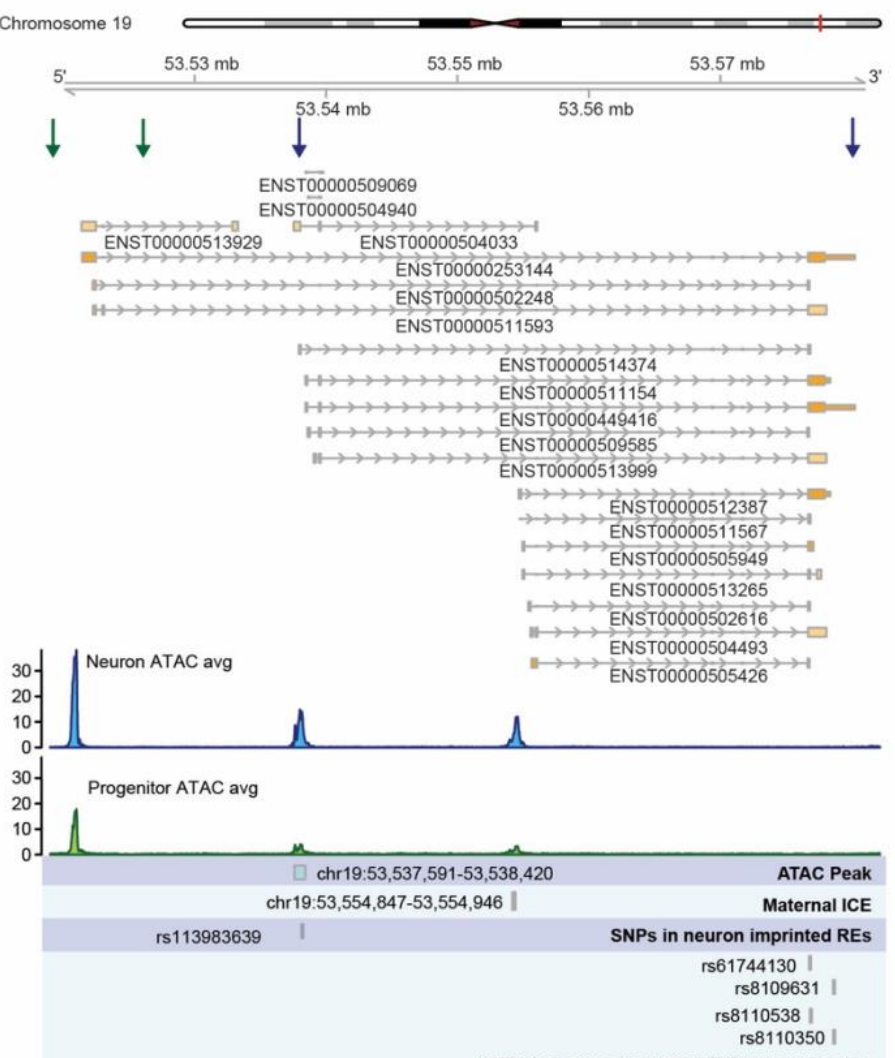

SNPs in neuron imprinted ZNF331 expression
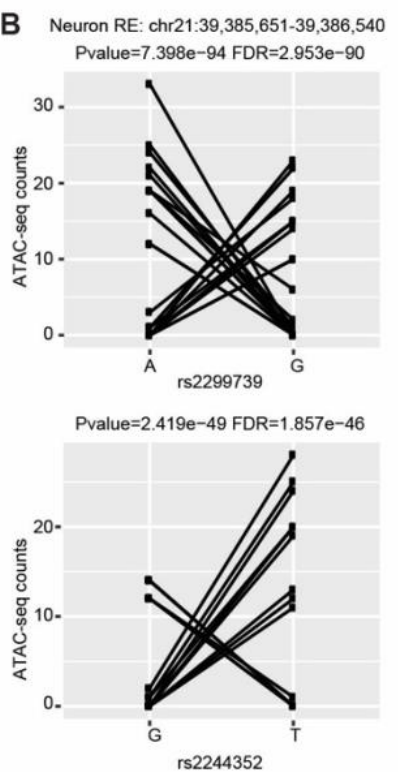

E Neuron RE: chr19:53,537,591-53,538,420 Pvalue $=1.184 \mathrm{e}-30 \mathrm{FDR}=2.715 \mathrm{e}-28$

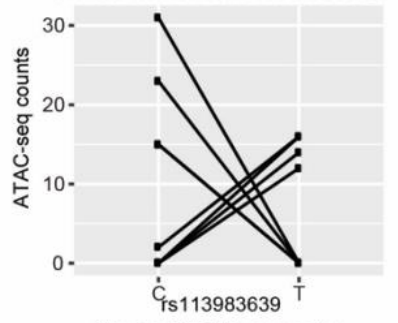

Neuron ZNF331 expression

Pvalue $=5.137 \mathrm{e}-247$ FDR $=1.622 \mathrm{e}-244$

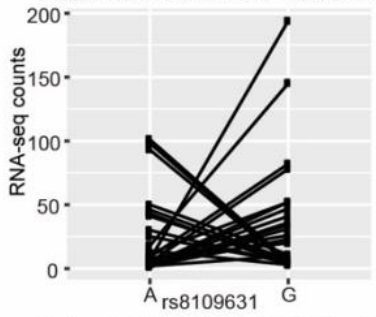

Pvalue $=2.562 \mathrm{e}-178$ FDR $=5.521 \mathrm{e}-176$

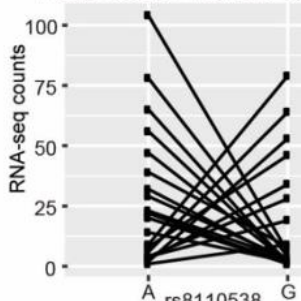

$A_{\text {rs } 8110538}$ 
Figure 4. Imprinted REs and genes indicating isoform-specific imprinting in neurons. (A) Coverage plot of ATAC-seq in neurons and progenitors at GET1 locus. (B) Allelic ATAC-seq counts for the SNPs in the imprinted RE in (A). (C) Methylation ratio in human fetal brain and 12 year old brain (Lister et al. 2013). (D) Coverage plot of ATAC-seq in neurons and progenitors at the ZNF331 locus. Boundaries of ZNF331 isoforms showed neuron-specific imprinted expression patterns are indicated by blue arrows. Boundaries of ZNF331 imprinted isoforms in LCL are indicated by red arrows. (C) Allelic ATAC/RNA-seq counts for SNPs in the imprinted RE and gene in (D).

\section{Progenitor-specific DLK1 imprinting at Kagami Ogata/Temple syndrome paternal uniparental disomy locus}

Uniparental disomy (UPD) results from homologous chromosomes, or parts of chromosomes, being inherited from only one parent (Robinson 2000). UPD and copy number variation mimicking UPD at imprinted sites results in abnormal expression. Copy number variation in the $14 q 32$ imprinted gene cluster can lead to distinct maternal or paternal UPD phenotypes, named Temple syndrome and Kagami Ogata syndrome, respectively (Beygo et al. 2015; Buiting et al. 2008; Chen et al. 2005; Kagami et al. 2008). Individuals with Temple syndrome (UPD(14)mat) have characteristic features including pre- and postnatal growth retardation and developmental delay (loannides et al. 2014). Kagami Ogata syndrome (UPD(14)pat) results in prenatal overgrowth, developmental delay, and facial abnormalities with full cheeks and protruding philtrum (Ogata and Kagami 2016; Rosenfeld et al. 2015). Maternal deletions in the genomic region containing maternally expressed genes MEG3, MEG8 and RTL1 and sometimes containing paternally expressed gene DLK1 have been identified in individuals with Kagami Ogata syndrome (Rosenfeld et al. 2015). Generally, individuals with maternal deletions in this region lack expression of the maternally expressed genes, but show overexpression of DLK1 in blood and placenta (Ogata and Kagami 2016). In Temple syndrome, DLK1 expression is lost due to the paternal deletion of this locus (Prasasya et al. 2020). We found evidence for imprinting of DLK1 gene expression in progenitors but not neurons (Figure 5C and 5D; Supplemental Figure 2E). 
$D L K 1$, previously known to be paternally expressed, promotes neurogenesis of neural progenitors in both mouse and human (Surmacz et al. 2012; Ferrón et al. 2011). In agreement with the function of $D L K 1$, we found $D L K 1$ showed a significantly higher expression level in progenitors than in neurons $(\log 2 F C=-3.67, F D R=6.5 e-244)$. Here, we suggest that $D L K 1$ imprinting in progenitors contributes to the opposing phenotypes of overgrowth observed in Kagami Ogata syndrome, and growth retardation observed in Temple syndrome. 
Figure 5

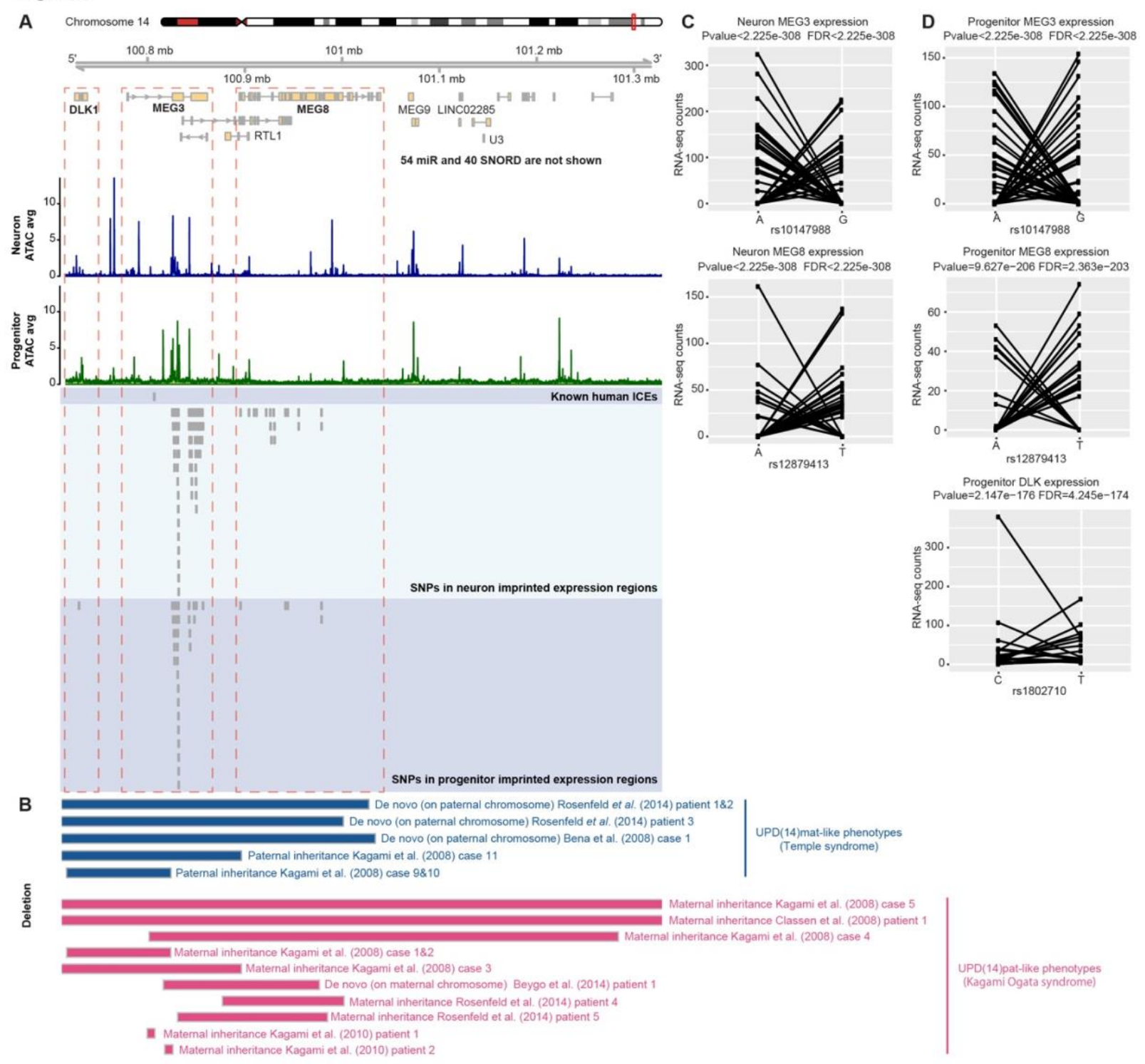

Figure 5. Progenitor-specific DLK1 imprinting at Kagami Ogata syndrome paternal uniparental disomy locus. (A) Coverage plot of ATAC-seq in neurons and progenitors at Kagami Ogata syndrome paternal uniparental disomy locus. DLK1 showed progenitorspecific imprinting expressions at genomic deletion regions. (B) Copy number variation in the 14q32 imprinted gene cluster related to Temple syndrome and Kagami Ogata syndrome. (C) Allelic RNA-seq counts for the SNPs in MEG3 (rs10147988) and MEG8 (rs12879413) in neurons. (D) Allelic RNA-seq counts for the SNPs in MEG3 (rs10147988), MEG8 (rs12879413), and $D L K 1$ in progenitors (rs1802710). 


\section{DISCUSSION}

Mutations at imprinted loci can lead to parent-of-origin dependent inheritance for neurodevelopmental disorders. In order to better explain the mechanism underlying parent-oforigin disorders, imprinting must be detected within relevant cell types. By combining highthroughput sequencing data (RNA-seq and ATAC-seq) with genotype data, we identified cell-type specific IREs and imprinted genes in two major cell types during human neuronal differentiation. We identified well-known IREs and genes in the PWS/AS region in neurons, providing confidence in our approach. We also identified cell-type specific IREs and genes as new candidates for genomic imprinting. We found cell-type specific REs may affect isoform-specific gene expression, as in neurons for GET1. Finally, we show that progenitor-specific imprinting of $D L K 1$ overlaps with deletions causing Kagami Ogata syndrome, suggesting neuronal progenitors contribute to neurobehavioral and growth changes observed in individuals with this syndrome.

Cell-type specific imprinted gene expression and chromatin accessibility showed dynamic changes of genomic imprinting during human neuronal differentiation. In addition to imprinted genes, we also identified IREs in neurons and progenitors, allowing us to explore the regulatory mechanisms of genomic imprinting. We found some genes only showed cell-type specific imprinted promoters but not imprinted expression, suggesting that the IREs are established prior to imprinted expression. We also found the IREs showed higher GC content and higher overlap with CpG islands, suggesting methylation of these REs is likely the regulatory mechanism underlying the imprinted signal in chromatin accessibility.

Although previous studies showed loss of imprinting in human pluripotent stem cells cultured in vitro (Bar et al. 2017; Frost et al. 2011), another study on induced pluripotent stem cells suggested genomic imprinting is not erased at the AS/PWS locus (Chamberlain et al. 2010). In this study, 
the well-known imprinted genes and promoters at AS/PWS locus were found in the neurons derived from neural progenitor cells in this study (Figure 2). The identification of these well-known imprinted genes indicated that the primary cell culture system used here was sufficient to study genomic imprinting at certain loci. However, it is unknown whether loss of imprinting at other sites occurred due to cell culture conditions.

Here, we inferred genomic imprinting using allelic ratios of RNA-seq and ATAC-seq reads. We separated genomic imprinting from allelic effects using a threshold for the number of individuals with extreme allelic ratio. A difficulty of identifying genomic imprinting using next generation sequencing data without genotype data from parents is to separate RMAE/RMACA from imprinted genes/REs. RMAE are mostly found in chromosome $\mathrm{X}$ due to the $\mathrm{X}$-chromosome inactivity, and there are less than 5\% of genes showing RMAE on autosomes (Kravitz and Gregg 2019). In this study, we removed the previously known RMAE/RMACA (Xu et al. 2017; Gimelbrant et al. 2007) to increase the confidence of imprinting calls. However, parental genotype data are necessary to completely disambiguate RMAE/RMACA from imprinting and so we refer to all novel imprinted genes/REs as putatively imprinted.

Additional experimental validation is also necessary to detect the molecular mechanisms of genomic imprinting during human neuronal differentiation. Genomic editing (deletion or modification) for IREs can be used to study their regulation of imprinting. Finally, we envision that combining the cell-type specific genomic imprinting with well-powered parent-of-origin genomewide association studies (GWASs) or parent-of-origin rare variant association studies, will allow a better understanding of parent-of-origin effects on neurodevelopmental disorders. 


\section{METHODS}

\section{Cell culture of primary human neural progenitor cells (phNPCs):}

We cultured and differentiated the phNPCs into neurons following the same methods in our previous work (Stein et al. 2014; Liang et al. 2021).

\section{ATAC-seq and RNA-seq library preparation for human neural progenitors and neurons}

ATAC-seq libraries were prepared immediately following cellular dissociation described in our previous methods (Buenrostro et al. 2015; Liang et al. 2021). All libraries were sequenced on an Illumina HiSeq2500 or MiSeq machine using 50 bp paired-end sequencing. RNA-seq libraries were prepared as previously described (Aygün et al. 2021) and were sequenced on a NovaSeq S2 flow cell using 150 bp paired-end sequencing.

\section{ATAC-seq, RNA-seq, and genotype data pre-processing}

Raw ATAC-seq and RNA-seq data were quality controlled and aligned to the human genome (GRCh38/hg38) using WASP to prevent mapping bias as previously described (Liang et al. 2021; Aygün et al. 2021). Genotype data were preprocessed and imputed as previously described (Liang et al. 2021).

\section{Allele-specific read counts}

We used GATK tools (McKenna et al. 2010) to extract allele-specific read counts for every biallelic SNP (in accessible peaks or expression regions). We first filtered for SNPs within each donor that had sufficient read depth by retaining SNPs with total counts greater than or equal to 10 for neuron and progenitor samples, separately. Then to calculate allelic imbalance in chromatin accessibility and gene expression, we retained those SNPs with average read counts for all 
heterozygous donors greater than or equal to 15 for chromatin accessibility and 30 for gene expression. Finally, we retained only those SNPs that meet these previous thresholds for at least 5 heterozygous donors.

\section{Estimation of over-dispersion and identification of imprinted chromatin accessibility and gene expression}

We identified over-dispersion using a likelihood ratio test based on the beta-binomial distribution for the allelic count (\# reads from the reference allele given the \# reads from the reference allele and alternative allele) at each SNPs. The allelic count can be modelled by a beta-binomial distribution, with the probability of expressing the parental-specific allele modelled by beta distribution (accounting for over-dispersion), and the number of reads observed modeled by a binomial distribution. The apeglm Bioconductor package was used to estimate parameters (Zitovsky and Love 2019). The likelihood ratio test was used to determine significance for the over-dispersion parameter for the heterozygous SNPs. To identify the imprinted chromatin accessibility and gene expression, we identify the SNPs in imprinted accessible or expression regions using the following three conditions: 1) SNPs have at least 2 donors with an allelic ratio greater than or equals to $0.8 ; 2$ ) SNPs have at least 2 donors with an allelic ratio less than or equals to 0.2 ; and 3 ) SNPs have significant over-dispersion of the allelic ratio (FDR $<0.05$ (Benjamini and Hochberg 1995)).

SNPs in the RMAE gene body and promoter regions were removed (Gimelbrant et al. 2007). For RMACA from mouse cells (Xu et al. 2017), we converted the RMACA regions from mouse genome (mm9) to the human genome (hg38) using liftOver from UCSCtools via the R package (rtracklayer v1.44.0).

\section{TFBS enrichment analysis}


Potential transcription factor binding sites were called in the human genome using TFBSTools from the JASPAR2016 core database as previously (Liang et al. 2021). We calculated the enrichment of TFBS in Neuron/Progenitor IREs using the binomial test (McLean et al. 2010). First, we found accessible regions ( $n$ ) overlapping with all TFBSs for a given TF and calculated the fraction of base pairs of the motif compared to the overall base pairs of accessible peaks (p). Then, we counted the number of IREs $(k)$ overlapping with TFBSs for this TF $(k)$. The final step was to calculate $P=\operatorname{Pr}_{\text {binom }}(x>=k \mid n, p)$ using the binomial test to get the significance of the enrichment. We further filtered the enrichment results by differential expression from the same set of cells, and only kept the TFs with cell-type specific significantly enriched in imprinted REs and significantly differentially expressed in the cell type (Aygün et al. 2021).

\section{Identification of differential methylation regions among sperms, oocytes and morula}

The genome-wide DNA methylation profiles for sperms $(\mathrm{N}=4)$, oocytes $(\mathrm{N}=2)$ and morula $(\mathrm{N}=3)$ were downloaded via NCBI Gene Expression Omnibus (GEO) under accession number GSE49828. R package 'MethylKit' (v1.16.1) was used to analyze differential methylation regions for sperms vs oocytes and morula or oocytes vs sperm and morula. After filtering the bases with $<5$ reads, 100-bp-tiles was called and the methylation level was estimated as previously described (Akalin et al. 2012). A logistic regression model is used to identify the differential methylation tiles. The $p$ values were adjusted to $q$ values by the SLIM method $(H .-Q$. Wang, Tuominen, and Tsai 2011). Tiles with q-value $<0.05$ and percent methylation difference larger than $25 \%$ were assigned as differential methylation regions. 
DATA ACCESS (including public database accession numbers for all newly generated data and/or reviewer links to deposited data when accessions are not yet public.

Previously published accessions should be included in the Methods section where appropriate)

ATAC-seq/RNA-seq data and genotype data for neurons and progenitors are available via dbGAP (ph001958 and phs2493).

Human CpG island:

https://genome.ucsc.edu/cgi-

bin/hgTables?hgsid=578954849_wF1QP81SIHdfr8b0kmZUOcsZcHYr\&clade=mammal\&org=Hu man\&db=hg38\&hgta_group=regulation\&hgta_track=knownGene\&hgta_table=0\&hgta_regionTy

pe=genome $\&$ position $=$ chr9\%3A133252000 -

133280861\&hgta_outputType=primaryTable\&hgta_outFileName=

Genome-wide DNA methylation profiles for sperms, oocytes and morula:

https://www.ncbi.nlm.nih.gov/geo/query/acc.cgi?acc=GSE49828

Imprinted genes:

https://geneimprint.com/site/genes-by-species

Genome-wide DNA methylation profiles for brain tissues:

https://www.ncbi.nlm.nih.gov/geo/query/acc.cgi?acc=GSE47966

\section{ACKNOWLEDGMENTS}

This work was supported by NIH (R00MH102357, U54EB020403, R01MH118349,

R01MH120125), and Brain Research Foundation to JLS.

\section{Author Contributions}

JLS and MIL conceived the study. JLS directed and supervised the study. JLS provided funding. NA performed pre-processing of allele specific gene expression data. MIL assisted with statistical identification of imprinting REs and genes. FI assisted with interpretation of the results. DL 
bioRxiv preprint doi: https://doi.org/10.1101/2021.10.04.463060; this version posted October 12, 2021. The copyright holder for this preprint (which was not certified by peer review) is the author/funder, who has granted bioRxiv a license to display the preprint in perpetuity. It is made available under aCC-BY 4.0 International license.

performed pre-processing of ATAC-seq data and identification of imprinting REs and genes. JLS and DL wrote the manuscript. All authors commented on and approved the final version of the manuscript.

\section{DISCLOSURE DECLARATION (including any conflicts of interest)}

The authors do not declare any conflicts of interest. 


\section{Supplementary Tables:}

Table S1. Imprinted REs in neurons and progenitors. VariantID is the name of the caSNP. RefAllele and altAllele are reference allele and alternative allele of the caSNP. Theta.hat is the estimate of dispersion using the apeglm Bioconductor package's bbEstDisp function (larger theta indicating less variance), p.hat and p.hat0 are the fitted allelic ratio for the full and reduced models respectively, and stat is the likelihood ratio test statistic. P.Value is the nominal $p$-value for the test of overdispersion; adj.P.Val is the Benjamini-Hochberg FDR adjusted p-value. H_Het_donor is the number of heterozygous donors for the SNP. counts are the total counts for the SNPs across all donors. Chr, peakstart and peakend are the coordinates (hg38) for the REs. Promotor represents the overlapped promoters with the RE. KnownImp if true means the RE overlaps with the promoter of known imprinted genes. The NeuronImprintedREs tab is for nIREs and the ProgenitorlmprintedREs tab is for pIREs.

Table S2. Imprinted genes in neurons and progenitors. SNPIDs is the name of the caSNP. RefAllele and altAllele are reference allele and alternative allele of the caSNP. Theta.hat, p.hat, p.hat0 and stats are as defined for Supplementary Table 1. P.Value is the nominal $p$-value of dispersion; adj.P.Val is the Benjamini-Hochberg FDR adjusted p-value. H_Het_donor is the number of heterozygous donors for the SNP. counts are the total counts for the SNPs across all donors. Gene is the gene name. Knownlmp if true means the gene is a known imprinted gene. The NeuronImprintedGenes tab is for neuron imprinted genes and the ProgenitorlmprintedGenes tab is for progenitor imprinted genes. 


\section{REFERENCES}

Akalin, Altuna, Matthias Kormaksson, Sheng Li, Francine E. Garrett-Bakelman, Maria E. Figueroa, Ari Melnick, and Christopher E. Mason. 2012. "methylKit: A Comprehensive R Package for the Analysis of Genome-Wide DNA Methylation Profiles." Genome Biology 13 (10): R87.

Alves da Silva, Antônio Francisco, Filipe Brum Machado, Érika Cristina Pavarino, Joice Matos Biselli-Périco, Bruna Lancia Zampieri, Ronaldo da Silva Francisco Junior, Pedro Thyago Mozer Rodrigues, et al. 2016. "Trisomy 21 Alters DNA Methylation in Parent-of-OriginDependent and -Independent Manners." PloS One 11 (4): e0154108.

Andergassen, Daniel, Christoph P. Dotter, Daniel Wenzel, Verena Sigl, Philipp C. Bammer, Markus Muckenhuber, Daniela Mayer, et al. 2017. "Mapping the Mouse Allelome Reveals Tissue-Specific Regulation of Allelic Expression." eLife 6 (August). https://doi.org/10.7554/eLife.25125.

Arnaud, Philippe. 2010. "Genomic Imprinting in Germ Cells: Imprints Are under Control." Reproduction 140 (3): 411-23.

Aygün, Nil, Angela L. Elwell, Dan Liang, Michael J. Lafferty, Kerry E. Cheek, Kenan P. Courtney, Jessica Mory, et al. 2021. "Brain-Trait-Associated Variants Impact Cell-TypeSpecific Gene Regulation during Neurogenesis." American Journal of Human Genetics, August. https://doi.org/10.1016/j.ajhg.2021.07.011.

Babak, Tomas, Brian Deveale, Christopher Armour, Christopher Raymond, Michele A. Cleary, Derek van der Kooy, Jason M. Johnson, and Lee P. Lim. 2008. "Global Survey of Genomic Imprinting by Transcriptome Sequencing." Current Biology: CB 18 (22): 1735-41.

Babak, Tomas, Brian DeVeale, Emily K. Tsang, Yiqi Zhou, Xin Li, Kevin S. Smith, Kim R. Kukurba, et al. 2015. "Genetic Conflict Reflected in Tissue-Specific Maps of Genomic Imprinting in Human and Mouse." Nature Genetics 47 (5): 544-49.

Baran, Yael, Meena Subramaniam, Anne Biton, Taru Tukiainen, Emily K. Tsang, Manuel A. Rivas, Matti Pirinen, et al. 2015. "The Landscape of Genomic Imprinting across Diverse Adult Human Tissues." Genome Research 25 (7): 927-36.

Barlow, Denise P., and Marisa S. Bartolomei. 2014. "Genomic Imprinting in Mammals." Cold Spring Harbor Perspectives in Biology 6 (2). https://doi.org/10.1101/cshperspect.a018382.

Bar, Shiran, Maya Schachter, Talia Eldar-Geva, and Nissim Benvenisty. 2017. "Large-Scale Analysis of Loss of Imprinting in Human Pluripotent Stem Cells." Cell Reports 19 (5): 95768.

Ben-David, Eyal, Shahar Shohat, and Sagiv Shifman. 2014. "Allelic Expression Analysis in the Brain Suggests a Role for Heterogeneous Insults Affecting Epigenetic Processes in Autism Spectrum Disorders." Human Molecular Genetics 23 (15): 4111-24.

Benjamini, Yoav, and Yosef Hochberg. 1995. "Controlling the False Discovery Rate: A Practical and Powerful Approach to Multiple Testing." Journal of the Royal Statistical Society: Series $B$ (Methodological). https://doi.org/10.1111/j.2517-6161.1995.tb02031.x.

Beygo, Jasmin, Miriam Elbracht, Karel de Groot, Matthias Begemann, Deniz Kanber, Konrad Platzer, Gabriele Gillessen-Kaesbach, et al. 2015. "Novel Deletions Affecting the MEG3DMR Provide Further Evidence for a Hierarchical Regulation of Imprinting in 14q32." European Journal of Human Genetics: EJHG 23 (2): 180-88.

Blagitko, N., S. Mergenthaler, U. Schulz, H. A. Wollmann, W. Craigen, T. Eggermann, H. H. Ropers, and V. M. Kalscheuer. 2000. "Human GRB10 Is Imprinted and Expressed from the Paternal and Maternal Allele in a Highly Tissue- and Isoform-Specific Fashion." Human Molecular Genetics 9 (11): 1587-95.

Bonthuis, Paul J., Wei-Chao Huang, Cornelia N. Stacher Hörndli, Elliott Ferris, Tong Cheng, and Christopher Gregg. 2015. "Noncanonical Genomic Imprinting Effects in Offspring." Cell 
Reports 12 (6): 979-91.

Bouschet, Tristan, Emeric Dubois, Christelle Reynès, Satya K. Kota, Stéphanie Rialle,

Stéphanie Maupetit-Méhouas, Mikael Pezet, et al. 2017. "In Vitro Corticogenesis from

Embryonic Stem Cells Recapitulates the In Vivo Epigenetic Control of Imprinted Gene

Expression." Cerebral Cortex 27 (3): 2418-33.

Brandler, William M., Danny Antaki, Madhusudan Gujral, Morgan L. Kleiber, Joe Whitney, Michelle S. Maile, Oanh Hong, et al. 2018. "Paternally Inherited Cis-Regulatory Structural

Variants Are Associated with Autism." Science 360 (6386): 327-31.

Buenrostro, Jason D., Beijing Wu, Howard Y. Chang, and William J. Greenleaf. 2015. "ATAC-

Seq: A Method for Assaying Chromatin Accessibility Genome-Wide." Current Protocols in

Molecular Biology / Edited by Frederick M. Ausubel ... [et Al.] 109 (January): 21.29.1-9.

Buiting, Karin, Deniz Kanber, José I. Martín-Subero, Wolfgang Lieb, Paulien Terhal, Beate

Albrecht, Sabine Purmann, et al. 2008. "Clinical Features of Maternal Uniparental Disomy

14 in Patients with an Epimutation and a Deletion of the Imprinted DLK1/GTL2 Gene Cluster." Human Mutation 29 (9): 1141-46.

Castel, Stephane E., Ami Levy-Moonshine, Pejman Mohammadi, Eric Banks, and Tuuli Lappalainen. 2015. "Tools and Best Practices for Data Processing in Allelic Expression Analysis." Genome Biology 16 (September): 195.

Chamberlain, Stormy J., Pin-Fang Chen, Khong Y. Ng, Fany Bourgois-Rocha, Fouad LemtiriChlieh, Eric S. Levine, and Marc Lalande. 2010. "Induced Pluripotent Stem Cell Models of the Genomic Imprinting Disorders Angelman and Prader-Willi Syndromes." Proceedings of the National Academy of Sciences of the United States of America 107 (41): 17668-73.

Chen, Chih-Ping, Schu-Rern Chern, Shuan-Pei Lin, Chyi-Chyang Lin, Yueh-Chun Li, Tzu-Hao Wang, Chen-Chi Lee, Chen-Wen Pan, Lie-Jiau Hsieh, and Wayseen Wang. 2005. "A Paternally Derived Inverted Duplication of Distal $14 q$ with a Terminal $14 q$ Deletion." American Journal of Medical Genetics. Part A 139A (2): 146-50.

Court, Franck, Chiharu Tayama, Valeria Romanelli, Alex Martin-Trujillo, Isabel Iglesias-Platas, Kohji Okamura, Naoko Sugahara, et al. 2014. "Genome-Wide Parent-of-Origin DNA Methylation Analysis Reveals the Intricacies of Human Imprinting and Suggests a Germline Methylation-Independent Mechanism of Establishment." Genome Research 24 (4): 554-69.

Cowley, Michael, David A. Skaar, Dereje D. Jima, Rachel L. Maguire, Kathleen M. Hudson, Sarah S. Park, Patricia Sorrow, and Cathrine Hoyo. 2018. "Effects of Cadmium Exposure on DNA Methylation at Imprinting Control Regions and Genome-Wide in Mothers and Newborn Children." Environmental Health Perspectives 126 (3): 037003.

Crowley, James J., Vasyl Zhabotynsky, Wei Sun, Shunping Huang, Isa Kemal Pakatci, Yunjung Kim, Jeremy R. Wang, et al. 2015. "Analyses of Allele-Specific Gene Expression in Highly Divergent Mouse Crosses Identifies Pervasive Allelic Imbalance." Nature Genetics 47 (4): 353-60.

Daelemans, Caroline, Matthew E. Ritchie, Guillaume Smits, Sayeda Abu-Amero, Ian M. Sudbery, Matthew S. Forrest, Susana Campino, et al. 2010. "High-Throughput Analysis of Candidate Imprinted Genes and Allele-Specific Gene Expression in the Human Term Placenta." BMC Genetics 11 (April): 25.

Dindot, Scott V., Barbara A. Antalffy, Meenakshi B. Bhattacharjee, and Arthur L. Beaudet. 2008. "The Angelman Syndrome Ubiquitin Ligase Localizes to the Synapse and Nucleus, and Maternal Deficiency Results in Abnormal Dendritic Spine Morphology." Human Molecular Genetics 17 (1): 111-18.

Docherty, Louise E., Faisal I. Rezwan, Rebecca L. Poole, Hannah Jagoe, Hannah Lake, Gabrielle A. Lockett, Hasan Arshad, et al. 2014. "Genome-Wide DNA Methylation Analysis of Patients with Imprinting Disorders Identifies Differentially Methylated Regions Associated with Novel Candidate Imprinted Genes." Journal of Medical Genetics 51 (4): 229-38.

Duffié, Rachel, Sophie Ajjan, Maxim V. Greenberg, Natasha Zamudio, Martin Escamilla del 
Arenal, Julian Iranzo, Ikuhiro Okamoto, Sandrine Barbaux, Patricia Fauque, and Déborah Bourc'his. 2014. "The Gpr1/Zdbf2 Locus Provides New Paradigms for Transient and Dynamic Genomic Imprinting in Mammals.” Genes \& Development 28 (5): 463-78.

Ferguson-Smith, Anne C. 2011. "Genomic Imprinting: The Emergence of an Epigenetic Paradigm." Nature Reviews. Genetics 12 (8): 565-75.

Ferrón, Sacri R., Marika Charalambous, Elizabeth Radford, Kirsten McEwen, Hendrik Wildner, Eleanor Hind, Jose Manuel Morante-Redolat, et al. 2011. "Postnatal Loss of Dlk1 Imprinting in Stem Cells and Niche Astrocytes Regulates Neurogenesis." Nature 475 (7356): 381-85.

Frost, Jennifer, Dave Monk, Dafni Moschidou, Pascale V. Guillot, Philip Stanier, Stephen L. Minger, Nicholas M. Fisk, Harry D. Moore, and Gudrun E. Moore. 2011. "The Effects of Culture on Genomic Imprinting Profiles in Human Embryonic and Fetal Mesenchymal Stem Cells." Epigenetics: Official Journal of the DNA Methylation Society 6 (1): 52-62.

Gimelbrant, Alexander, John N. Hutchinson, Benjamin R. Thompson, and Andrew Chess. 2007. "Widespread Monoallelic Expression on Human Autosomes." Science 318 (5853): 113640.

Gregg, Christopher, Jiangwen Zhang, Brandon Weissbourd, Shujun Luo, Gary P. Schroth, David Haig, and Catherine Dulac. 2010. "High-Resolution Analysis of Parent-of-Origin Allelic Expression in the Mouse Brain." Science 329 (5992): 643-48.

Grütz, Karen, Philip Seibler, Anne Weissbach, Katja Lohmann, Francesca A. Carlisle, Derek J. Blake, Ana Westenberger, Christine Klein, and Anne Grünewald. 2017. "Faithful SGCE Imprinting in iPSC-Derived Cortical Neurons: An Endogenous Cellular Model of MyoclonusDystonia." Scientific Reports 7 (February): 41156.

GTEx Consortium. 2020. "The GTEx Consortium Atlas of Genetic Regulatory Effects across Human Tissues." Science 369 (6509): 1318-30.

Guo, Hongshan, Ping Zhu, Liying Yan, Rong Li, Boqiang Hu, Ying Lian, Jie Yan, et al. 2014. "The DNA Methylation Landscape of Human Early Embryos." Nature 511 (7511): 606-10.

Hanna, C. W., and G. Kelsey. 2014. "The Specification of Imprints in Mammals." Heredity 113 (2): 176-83.

Ho-Shing, Olivia, and Catherine Dulac. 2019. "Influences of Genomic Imprinting on Brain Function and Behavior." Current Opinion in Behavioral Sciences 25 (February): 66-76.

Hsiao, Jack S., Noelle D. Germain, Andrea Wilderman, Christopher Stoddard, Luke A. Wojenski, Geno J. Villafano, Leighton Core, Justin Cotney, and Stormy J. Chamberlain. 2019. "A Bipartite Boundary Element Restricts UBE3A Imprinting to Mature Neurons." Proceedings of the National Academy of Sciences of the United States of America 116 (6): 2181-86.

Huntriss, John D., Karen E. Hemmings, Matthew Hinkins, Anthony J. Rutherford, Roger G. Sturmey, Kay Elder, and Helen M. Picton. 2013. "Variable Imprinting of the MEST Gene in Human Preimplantation Embryos." European Journal of Human Genetics: EJHG 21 (1): 40-47.

loannides, Yiannis, Kemi Lokulo-Sodipe, Deborah J. G. Mackay, Justin H. Davies, and I. Karen Temple. 2014. "Temple Syndrome: Improving the Recognition of an Underdiagnosed Chromosome 14 Imprinting Disorder: An Analysis of 51 Published Cases." Journal of Medical Genetics 51 (8): 495-501.

Ishida, Miho, and Gudrun E. Moore. 2013. "The Role of Imprinted Genes in Humans." Molecular Aspects of Medicine 34 (4): 826-40.

Jadhav, Bharati, Ramin Monajemi, Kristina K. Gagalova, Daniel Ho, Harmen H. M. Draisma, Mark A. van de Wiel, Lude Franke, et al. 2019. "RNA-Seq in 296 Phased Trios Provides a High-Resolution Map of Genomic Imprinting." BMC Biology 17 (1): 50.

Jay, P., C. Rougeulle, A. Massacrier, A. Moncla, M. G. Mattei, P. Malzac, N. Roëckel, et al. 1997. "The Human Necdin Gene, NDN, Is Maternally Imprinted and Located in the PraderWilli Syndrome Chromosomal Region." Nature Genetics 17 (3): 357-61. 
Kagami, Masayo, Yoichi Sekita, Gen Nishimura, Masahito Irie, Fumiko Kato, Michiyo Okada, Shunji Yamamori, et al. 2008. "Deletions and Epimutations Affecting the Human 14q32.2 Imprinted Region in Individuals with Paternal and Maternal upd(14)-like Phenotypes." Nature Genetics. https://doi.org/10.1038/ng.2007.56.

Kosaki, K., R. Kosaki, W. J. Craigen, and N. Matsuo. 2000. "Isoform-Specific Imprinting of the Human PEG1/MEST Gene." American Journal of Human Genetics 66 (1): 309-12.

Kravitz, Stephanie N., and Christopher Gregg. 2019. "New Subtypes of Allele-Specific Epigenetic Effects: Implications for Brain Development, Function and Disease." Current Opinion in Neurobiology 59 (December): 69-78.

Laukoter, Susanne, Florian M. Pauler, Robert Beattie, Nicole Amberg, Andi H. Hansen, Carmen Streicher, Thomas Penz, Christoph Bock, and Simon Hippenmeyer. 2020. "Cell-Type Specificity of Genomic Imprinting in Cerebral Cortex." Neuron 107 (6): 1160-79.e9.

Liang, Dan, Angela L. Elwell, Nil Aygün, Oleh Krupa, Justin M. Wolter, Felix A. Kyere, Michael J. Lafferty, et al. 2021. "Cell-Type-Specific Effects of Genetic Variation on Chromatin Accessibility during Human Neuronal Differentiation." Nature Neuroscience, May. https://doi.org/10.1038/s41593-021-00858-w.

Lister, Ryan, Eran A. Mukamel, Joseph R. Nery, Mark Urich, Clare A. Puddifoot, Nicholas D. Johnson, Jacinta Lucero, et al. 2013. "Global Epigenomic Reconfiguration during Mammalian Brain Development.” Science 341 (6146): 1237905.

Llères, David, Benoît Moindrot, Rakesh Pathak, Vincent Piras, Mélody Matelot, Benoît Pignard, Alice Marchand, et al. 2019. "CTCF Modulates Allele-Specific Sub-TAD Organization and Imprinted Gene Activity at the Mouse Dlk1-Dio3 and Igf2-H19 Domains." Genome Biology $20(1): 272$.

Lopes, Susana, Annabelle Lewis, Petra Hajkova, Wendy Dean, Joachim Oswald, Thierry Forné, Adele Murrell, et al. 2003. "Epigenetic Modifications in an Imprinting Cluster Are Controlled by a Hierarchy of DMRs Suggesting Long-Range Chromatin Interactions." Human Molecular Genetics 12 (3): 295-305.

Lucifero, Diana, Carmen Mertineit, Hugh J. Clarke, Timothy H. Bestor, and Jacquetta M. Trasler. 2002. "Methylation Dynamics of Imprinted Genes in Mouse Germ Cells." Genomics 79 (4): 530-38.

Martins-Taylor, Kristen, Jack S. Hsiao, Pin-Fang Chen, Heather Glatt-Deeley, Adam J. De Smith, Alexandra I. F. Blakemore, Marc Lalande, and Stormy J. Chamberlain. 2014. "Imprinted Expression of UBE3A in Non-Neuronal Cells from a Prader-Willi Syndrome Patient with an Atypical Deletion." Human Molecular Genetics 23 (9): 2364-73.

McKenna, Aaron, Matthew Hanna, Eric Banks, Andrey Sivachenko, Kristian Cibulskis, Andrew Kernytsky, Kiran Garimella, et al. 2010. "The Genome Analysis Toolkit: A MapReduce Framework for Analyzing next-Generation DNA Sequencing Data." Genome Research 20 (9): 1297-1303.

McLean, Cory Y., Dave Bristor, Michael Hiller, Shoa L. Clarke, Bruce T. Schaar, Craig B. Lowe, Aaron M. Wenger, and Gill Bejerano. 2010. "GREAT Improves Functional Interpretation of Cis-Regulatory Regions." Nature Biotechnology 28 (5): 495-501.

Mozaffari, Sahar V., Jeanne M. DeCara, Sanjiv J. Shah, Carlo Sidore, Edoardo Fiorillo, Francesco Cucca, Roberto M. Lang, Dan L. Nicolae, and Carole Ober. 2019. "Parent-ofOrigin Effects on Quantitative Phenotypes in a Large Hutterite Pedigree." Communications Biology 2 (January): 28.

Nakabayashi, Kazuhiko, Alex Martin Trujillo, Chiharu Tayama, Cristina Camprubi, Wataru Yoshida, Pablo Lapunzina, Aurora Sanchez, et al. 2011. "Methylation Screening of Reciprocal Genome-Wide UPDs Identifies Novel Human-Specific Imprinted Genes." Human Molecular Genetics 20 (16): 3188-97.

Nicholls, R. D. 2000. "The Impact of Genomic Imprinting for Neurobehavioral and Developmental Disorders.” The Journal of Clinical Investigation 105 (4): 413-18. 
Nicholls, R. D., S. Saitoh, and B. Horsthemke. 1998. "Imprinting in Prader-Willi and Angelman Syndromes." Trends in Genetics: TIG 14 (5): 194-200.

Nowakowski, Tomasz J., Aparna Bhaduri, Alex A. Pollen, Beatriz Alvarado, Mohammed A. Mostajo-Radji, Elizabeth Di Lullo, Maximilian Haeussler, et al. 2017. "Spatiotemporal Gene Expression Trajectories Reveal Developmental Hierarchies of the Human Cortex." Science 358 (6368): 1318-23.

Ogata, Tsutomu, and Masayo Kagami. 2016. "Kagami-Ogata Syndrome: A Clinically Recognizable upd(14)pat and Related Disorder Affecting the Chromosome 14q32.2 Imprinted Region." Journal of Human Genetics. https://doi.org/10.1038/jhg.2015.113.

Ono, Ryuichi, Kenji Nakamura, Kimiko Inoue, Mie Naruse, Takako Usami, Noriko WakisakaSaito, Toshiaki Hino, et al. 2006. "Deletion of Peg10, an Imprinted Gene Acquired from a Retrotransposon, Causes Early Embryonic Lethality." Nature Genetics 38 (1): 101-6.

Oreper, Daniel, Sarah A. Schoenrock, Rachel McMullan, Robin Ervin, Joseph Farrington, Darla R. Miller, Fernando Pardo-Manuel de Villena, William Valdar, and Lisa M. Tarantino. 2018. "Reciprocal F1 Hybrids of Two Inbred Mouse Strains Reveal Parent-of-Origin and Perinatal Diet Effects on Behavior and Expression." G3 8 (11): 3447-68.

Paulsen, M., and A. C. Ferguson-Smith. 2001. "DNA Methylation in Genomic Imprinting, Development, and Disease." The Journal of Pathology 195 (1): 97-110.

Perez, Julio D., Nimrod D. Rubinstein, and Catherine Dulac. 2016. "New Perspectives on Genomic Imprinting, an Essential and Multifaceted Mode of Epigenetic Control in the Developing and Adult Brain." Annual Review of Neuroscience 39 (July): 347-84.

Perez, Julio D., Nimrod D. Rubinstein, Daniel E. Fernandez, Stephen W. Santoro, Leigh A. Needleman, Olivia Ho-Shing, John J. Choi, et al. 2015. "Quantitative and Functional Interrogation of Parent-of-Origin Allelic Expression Biases in the Brain." eLife 4 (July): e07860.

Perk, Jonathan, Kirill Makedonski, Laura Lande, Howard Cedar, Aharon Razin, and Ruth Shemer. 2002. "The Imprinting Mechanism of the Prader-Willi/Angelman Regional Control Center." The EMBO Journal 21 (21): 5807-14.

Plasschaert, Robert N., and Marisa S. Bartolomei. 2014. "Genomic Imprinting in Development, Growth, Behavior and Stem Cells." Development 141 (9): 1805-13.

Pólvora-Brandão, Duarte, Mariana Joaquim, Inês Godinho, Domenico Aprile, Ana Rita Álvaro, Isabel Onofre, Ana Cláudia Raposo, Luís Pereira de Almeida, Sofia T. Duarte, and Simão T. da Rocha. 2018. "Loss of Hierarchical Imprinting Regulation at the PraderWilli/Angelman Syndrome Locus in Human iPSCs." Human Molecular Genetics 27 (23): 3999-4011.

Prasasya, Rexxi, Kristen V. Grotheer, Linda D. Siracusa, and Marisa S. Bartolomei. 2020. "Temple Syndrome and Kagami-Ogata Syndrome: Clinical Presentations, Genotypes, Models and Mechanisms." Human Molecular Genetics 29 (R1): R107-16.

Prickett, Adam R., and Rebecca J. Oakey. 2012. "A Survey of Tissue-Specific Genomic Imprinting in Mammals." Molecular Genetics and Genomics: MGG 287 (8): 621-30.

Reinius, Björn, and Rickard Sandberg. 2015. "Random Monoallelic Expression of Autosomal Genes: Stochastic Transcription and Allele-Level Regulation." Nature Reviews. Genetics 16 (11): 653-64.

Robinson, W. P. 2000. "Mechanisms Leading to Uniparental Disomy and Their Clinical Consequences." BioEssays: News and Reviews in Molecular, Cellular and Developmental Biology 22 (5): 452-59.

Rosenfeld, Jill A., Joyce E. Fox, Maria Descartes, Fallon Brewer, Tracy Stroud, Jerome L. Gorski, Sheila J. Upton, et al. 2015. "Clinical Features Associated with Copy Number Variations of the 14q32 Imprinted Gene Cluster." American Journal of Medical Genetics. Part A 167A (2): 345-53.

Sanli, Ildem, and Robert Feil. 2015. "Chromatin Mechanisms in the Developmental Control of 
Imprinted Gene Expression." The International Journal of Biochemistry \& Cell Biology 67 (October): 139-47.

Santoni, Federico A., Georgios Stamoulis, Marco Garieri, Emilie Falconnet, Pascale Ribaux, Christelle Borel, and Stylianos E. Antonarakis. 2017. "Detection of Imprinted Genes by Single-Cell Allele-Specific Gene Expression." American Journal of Human Genetics 100 (3): 444-53.

Skelly, Daniel A., Marnie Johansson, Jennifer Madeoy, Jon Wakefield, and Joshua M. Akey. 2011. "A Powerful and Flexible Statistical Framework for Testing Hypotheses of AlleleSpecific Gene Expression from RNA-Seq Data." Genome Research 21 (10): 1728-37.

Stanurova, Jana, Anika Neureiter, Michaela Hiber, Hannah de Oliveira Kessler, Kristin Stolp, Roman Goetzke, Diana Klein, Agnes Bankfalvi, Hannes Klump, and Laura Steenpass. 2016. "Angelman Syndrome-Derived Neurons Display Late Onset of Paternal UBE3A Silencing." Scientific Reports 6 (August): 30792.

Stein, Jason L., Luis de la Torre-Ubieta, Yuan Tian, Neelroop N. Parikshak, Israel A. Hernández, Maria C. Marchetto, Dylan K. Baker, et al. 2014. "A Quantitative Framework to Evaluate Modeling of Cortical Development by Neural Stem Cells." Neuron 83 (1): 69-86.

Stelzer, Yonatan, Shiran Bar, Osnat Bartok, Shaked Afik, Daniel Ronen, Sebastian Kadener, and Nissim Benvenisty. 2015. "Differentiation of Human Parthenogenetic Pluripotent Stem Cells Reveals Multiple Tissue- and Isoform-Specific Imprinted Transcripts." Cell Reports 11 (2): 308-20.

Surmacz, Beata, Parinya Noisa, Jessica R. Risner-Janiczek, Kailyn Hui, Mark Ungless, Wei Cui, and Meng Li. 2012. "DLK1 Promotes Neurogenesis of Human and Mouse Pluripotent Stem Cell-Derived Neural Progenitors via Modulating Notch and BMP Signalling." Stem Cell Reviews and Reports 8 (2): 459-71.

Takahashi, Nozomi, Andrea Coluccio, Christian W. Thorball, Evarist Planet, Hui Shi, Sandra Offner, Priscilla Turelli, Michael Imbeault, Anne C. Ferguson-Smith, and Didier Trono. 2019. "ZNF445 Is a Primary Regulator of Genomic Imprinting." Genes \& Development 33 (1-2): 49-54.

Tinarelli, Federico, Celina Garcia-Garcia, Francesco Nicassio, and Valter Tucci. 2014. "Parentof-Origin Genetic Background Affects the Transcriptional Levels of Circadian and Neuronal Plasticity Genes Following Sleep Loss." Philosophical Transactions of the Royal Society of London. Series B, Biological Sciences 369 (1637): 20120471.

Vilardi, Fabio, Holger Lorenz, and Bernhard Dobberstein. 2011. "WRB Is the Receptor for TRC40/Asna1-Mediated Insertion of Tail-Anchored Proteins into the ER Membrane." Journal of Cell Science 124 (Pt 8): 1301-7.

Wang, Hong-Qiang, Lindsey K. Tuominen, and Chung-Jui Tsai. 2011. "SLIM: A Sliding Linear Model for Estimating the Proportion of True Null Hypotheses in Datasets with Dependence Structures." Bioinformatics 27 (2): 225-31.

Wang, Qianwei, Jacqueline Chow, Jenny Hong, Anne Ferguson Smith, Carol Moreno, Peter Seaby, Paul Vrana, et al. 2011. "Recent Acquisition of Imprinting at the Rodent Sfmbt2 Locus Correlates with Insertion of a Large Block of miRNAs." BMC Genomics 12 (April): 204.

Wang, X., and A. G. Clark. 2014. "Using next-Generation RNA Sequencing to Identify Imprinted Genes." Heredity 113 (2): 156-66.

Wang, Xu, Paul D. Soloway, and Andrew G. Clark. 2011. "A Survey for Novel Imprinted Genes in the Mouse Placenta by mRNA-Seq." Genetics 189 (1): 109-22.

Wang, Xu, Qi Sun, Sean D. McGrath, Elaine R. Mardis, Paul D. Soloway, and Andrew G. Clark. 2008. "Transcriptome-Wide Identification of Novel Imprinted Genes in Neonatal Mouse Brain." PLoS ONE. https://doi.org/10.1371/journal.pone.0003839.

Watrin, Françoise, Elodie Le Meur, Nathalie Roeckel, Marie-Anne Ripoche, Luisa Dandolo, and Françoise Muscatelli. 2005. "The Prader-Willi Syndrome Murine Imprinting Center Is Not 
Involved in the Spatio-Temporal Transcriptional Regulation of the Necdin Gene." BMC Genetics 6 (January): 1.

Wolter, Justin M., Hanqian Mao, Giulia Fragola, Jeremy M. Simon, James L. Krantz, Hannah O. Bazick, Baris Oztemiz, Jason L. Stein, and Mark J. Zylka. 2020. "Cas9 Gene Therapy for Angelman Syndrome Traps Ube3a-ATS Long Non-Coding RNA.” Nature 587 (7833): 28184.

Xu, Jin, Ava C. Carter, Anne-Valerie Gendrel, Mikael Attia, Joshua Loftus, William J. Greenleaf, Robert Tibshirani, Edith Heard, and Howard Y. Chang. 2017. "Landscape of Monoallelic DNA Accessibility in Mouse Embryonic Stem Cells and Neural Progenitor Cells." Nature Genetics 49 (3): 377-86.

Zhang, Xun, Yunli Zhou, Kshama R. Mehta, Daniel C. Danila, Staci Scolavino, Stacey R. Johnson, and Anne Klibanski. 2003. "A Pituitary-Derived MEG3 Isoform Functions as a Growth Suppressor in Tumor Cells." The Journal of Clinical Endocrinology and Metabolism 88 (11): 5119-26.

Zink, Florian, Droplaug N. Magnusdottir, Olafur T. Magnusson, Nicolas J. Walker, Tiffany J. Morris, Asgeir Sigurdsson, Gisli H. Halldorsson, et al. 2018. "Insights into Imprinting from Parent-of-Origin Phased Methylomes and Transcriptomes." Nature Genetics 50 (11): 154252.

Zitovsky, Joshua P., and Michael I. Love. 2019. "Fast Effect Size Shrinkage Software for BetaBinomial Models of Allelic Imbalance." F1000Research. https://doi.org/10.12688/f1000research.20916.1. 